\title{
Compactness properties of Feller semigroups
}

by

G. Metafune (Lecce), D. Pallara (Lecce) and M. Wacker (Tübingen)

Abstract. We study the compactness of Feller semigroups generated by second order elliptic partial differential operators with unbounded coefficients in spaces of continuous functions in $\mathbb{R}^{N}$.

1. Introduction. Given a second order elliptic differential operator

$$
A u(x):=\sum_{i, j=1}^{N} a_{i j}(x) D_{i j} u(x)+\sum_{i=1}^{N} b_{i}(x) D_{i} u(x), \quad x \in \mathbb{R}^{N},
$$

we consider the parabolic problem

$$
\begin{cases}D_{t} u(t, x)=A u(t, x), & t>0, x \in \mathbb{R}^{N}, \\ u(0, x)=f(x), & x \in \mathbb{R}^{N} .\end{cases}
$$

We assume that $a_{i j}=a_{j i}$, all the coefficients $a_{i j}, b_{i}$ are real-valued and belong to $C_{\text {loc }}^{\alpha}\left(\mathbb{R}^{N}\right)$, and the ellipticity condition

$$
\sum_{i, j=1}^{N} a_{i j}(x) \xi_{i} \xi_{j} \geq \nu(x)|\xi|^{2}, \quad \xi \in \mathbb{R}^{N}
$$

holds with $\inf _{K} \nu>0$ for every compact $K \subset \mathbb{R}^{N}$. The operator $A$ is locally uniformly elliptic, i.e., it is uniformly elliptic on every compact subset of $\mathbb{R}^{N}$; however it is not (globally) uniformly elliptic since we are assuming neither that $\nu$ is bounded away from 0 , nor that the coefficients are bounded.

In [22] we have presented the construction of a semigroup $(T(t))_{t \geq 0}$ which gives, for positive $f$, the minimal solution among all the positive solutions of (1.2). Even though this semigroup is not strongly continuous in $C_{\mathrm{b}}\left(\mathbb{R}^{N}\right)$, the function $u(t, x)=T(t) f(x)$ is a bounded classical solution of (1.2) such that $u(t, \cdot) \rightarrow f$ uniformly on compact sets as $t \rightarrow 0$. This solution is the unique bounded solution of (1.2) if and only if the operator $\lambda-A$ is injective on the maximal domain in $C_{\mathrm{b}}\left(\mathbb{R}^{N}\right)$

$$
D_{\max }(A):=\left\{u \in C_{\mathrm{b}}\left(\mathbb{R}^{N}\right) \cap W_{\text {loc }}^{2, p}\left(\mathbb{R}^{N}\right) \text { for all } p<\infty: A u \in C_{\mathrm{b}}\left(\mathbb{R}^{N}\right)\right\}
$$

2000 Mathematics Subject Classification: 35K65, 47D07, 60J35. 
for positive $\lambda$. On the other hand, $T(t) f$ gives for every $f \in C_{\mathrm{b}}\left(\mathbb{R}^{N}\right)$ a solution vanishing at infinity if and only if $\lambda-A$ is surjective from the Dirichlet domain

$$
D(A):=D_{\max }(A) \cap C_{0}\left(\mathbb{R}^{N}\right)
$$

onto $C_{\mathrm{b}}\left(\mathbb{R}^{N}\right)$ for $\lambda>0$.

The interest in elliptic operators with coefficients unbounded or vanishing at infinity comes both from the theory of partial differential equations and from diffusion problems in $\mathbb{R}^{N}$. We refer to [22] for more information and further references. Let us only point out that in the theory of diffusion processes the parabolic equation $D_{t} u=A u$ is the Kolmogorov backward equation, and describes by duality the evolution of the probability distribution, hence it is naturally studied in spaces of continuous functions. In the one-dimensional case a detailed theory has been developed in the 1950s by W. Feller (see [13]) who gave an explicit description of all the boundary conditions that can be added to the differential operator $A$ on a real interval $I$ so that it generates a positive and contractive semigroup in $C(\bar{I})$. Feller's results are expressed through a classification of the endpoints of $I$ (see Subsection 2.2 below) which in particular distinguishes between accessible and inaccessible boundaries, i.e., endpoints that can or cannot be reached by the particle subject to diffusion. An account of the higher-dimensional case is presented in [22], in an analytical setting.

Here, we are concerned with compactness properties of the resolvent operator and the semigroup. Compactness properties of elliptic operators in the whole of $\mathbb{R}^{N}$ are not shared by uniformly elliptic operators, and in the present case depend essentially on the growth of the coefficients at infinity. In order to give a heuristic explanation of our main results, let us consider the operator

$$
A u(x)=\Delta u(x)+\sum_{i=1}^{N} b_{i}(x) D_{i} u(x)
$$

with $b(x):=\left(b_{1}(x), \ldots, b_{N}(x)\right)$ depending only on $|x|$.

If $b(x) \cdot x \cong|x|^{2+\varepsilon}$ for some $\varepsilon>0$, then the drift points outwards and $\infty$ is accessible, that is, the paths of the underlying Markov process explode in a finite time, and the semigroup is generated (in a suitable sense) by $(A, D(A))$. Then $\infty$ acts as an absorbing boundary for the process which, after a change of scale, can be viewed as a process in a bounded set with Dirichlet boundary conditions. In this situation one expects compactness and, in fact, this is always the case (see Theorem 3.2) when the semigroup is generated by $(A, D(A))$.

On the contrary, if $b(x) \cdot x \cong-|x|^{2+\varepsilon}$ for some $\varepsilon>0$, then the strong inward drift given by $b$ forces the particle to stay with high probability in a 
compact set (depending on the time $t$ ), no matter what the initial position is. This yields again the compactness of the semigroup (see Theorem 3.12) which, in this situation, is generated by $\left(A, D_{\max }(A)\right)$.

Both results above are false if $\varepsilon=0$. For example, they fail in the case of Ornstein-Uhlenbeck operators (see [24], [21]).

When $\lambda-A$ is injective on $D_{\max }(A)$ our compactness results for the resolvent follow from those for the semigroup, by standard Laplace transform methods. However, we do not see how to prove them directly.

As the general discussion in [22], also the results presented here rely on the existence of suitable Lyapunov functions. This in turn is sometimes obtained through comparison with radial operators, which are essentially one-dimensional and allow us to apply Feller's theory. In Section 2 we recall from [22] the construction of the semigroup, the basics of Feller's theory we need and the construction of radial comparison operators. Section 3 is devoted to the proof of the main compactness results, and is divided into two subsections, corresponding to the cases where the semigroup is generated with the Dirichlet or the maximal domain. In Section 4 we draw some consequences of compactness, in the latter case, also in connection to the invariant measure associated with the semigroup. Section 5 is entirely devoted to the discussion of several examples.

Notation. For $x \in \mathbb{R}^{N},|x|$ denotes the euclidean norm, and $B_{\varrho}=$ $\left\{x \in \mathbb{R}^{N}:|x|<\varrho\right\}$ the open ball with radius $\varrho>0$. We use polar coordinates $r \geq 0, \omega \in \partial B_{1}$, so that $x=r \omega$. As regards function spaces, $L^{p}$ spaces are always meant with respect to the Lebesgue measure, unless otherwise specified, and are endowed with the usual norm $\|\cdot\|_{p}$. Moreover, $W^{k, p}(\Omega)$ is the Sobolev space of functions measurable in the open set $\Omega \subset \mathbb{R}^{N}$ which have weak derivatives $p$-summable in $\Omega$ up to order $k$, endowed with the usual norm $\|\cdot\|_{W^{k, p}(\Omega)}$. We set $u \in W_{\text {loc }}^{k, p}(\Omega)$ if $\varphi u \in W^{k, p}(\Omega)$ for every $\varphi \in C_{0}^{\infty}(\Omega)$. We denote by $C_{\mathrm{b}}\left(\mathbb{R}^{N}\right)$ the space of bounded continuous functions on $\mathbb{R}^{N}$, and by $C_{0}\left(\mathbb{R}^{N}\right), C_{0}\left(B_{\varrho}\right)$ the spaces

$$
\begin{aligned}
C_{0}\left(\mathbb{R}^{N}\right) & :=\left\{u \in C\left(\mathbb{R}^{N}\right): \lim _{|x| \rightarrow \infty} u(x)=0\right\}, \\
C_{0}\left(B_{\varrho}\right) & :=\left\{u \in C\left(\bar{B}_{\varrho}\right):\left.u\right|_{\partial B_{\varrho}}=0\right\},
\end{aligned}
$$

always endowed with the sup norm $\|\cdot\|_{\infty}$. We also deal with spaces of (locally) Hölder continuous functions: for $k \geq 0,0<\alpha \leq 1, C_{\text {loc }}^{k+\alpha}(\Omega)$ denotes the space of functions $k$ times continuously differentiable, with the $k$ th derivative Hölder continuous with exponent $\alpha$ in every compact subset of $\Omega$. For functions of $N+1$ variables $t \in] a, b\left[\subset \mathbb{R}, x \in \Omega \subset \mathbb{R}^{N}, f(t, x) \in\right.$ $C_{\text {loc }}^{1+\alpha / 2,2+\alpha}(] a, b[\times \Omega)$ means that $D_{t} f=\partial f / \partial t$ and $D_{i j} f=\partial^{2} f / \partial x_{i} \partial x_{j}$ are $\alpha$-Hölder continuous in every compact subset of $] a, b[\times \Omega$ with respect 
to the parabolic distance $d((t, x),(\tau, y)):=|t-\tau|^{1 / 2}+|x-y|$. Sometimes we shall use these spaces also with $\alpha=0$. In this case Hölder continuity is replaced by continuity. Finally, in the whole paper $\lambda$ is a positive parameter.

\section{Preliminaries}

2.1. Construction of $(T(t))_{t \geq 0}$. We briefly recall the construction of a semigroup $(T(t))_{t \geq 0}$ yielding a solution to problem (1.2). We refer the reader to [22] and the references therein for the proofs of the following results and further information.

Fix a ball $B_{\varrho}$. Since $A$ is uniformly elliptic on this ball, we obtain a unique solution of problem (1.2) in $B_{\varrho}$ with Dirichlet boundary conditions on $\partial B_{\varrho}$. In the language of semigroup theory, the operator $A$ with domain

$$
D_{\varrho}(A)=\left\{u \in C_{0}\left(B_{\varrho}\right) \cap W^{2, p}\left(B_{\varrho}\right) \text { for all } p<\infty: A u \in C\left(\bar{B}_{\varrho}\right)\right\}
$$

generates an analytic semigroup $\left(T_{\varrho}(t)\right)_{t \geq 0}$ of positive contractions in the space $C\left(\bar{B}_{\varrho}\right)$ and, for every $f \in C\left(\bar{B}_{\varrho}\right)$, the function $u_{\varrho}(t, x)=T_{\varrho}(t) f(x)$ satisfies

$$
\begin{cases}D_{t} u_{\varrho}=A u_{\varrho}, & t>0, x \in B_{\varrho}, \\ u_{\varrho}(t, x)=0, & t>0, x \in \partial B_{\varrho}, \\ u_{\varrho}(0, x)=f(x), & x \in \bar{B}_{\varrho} .\end{cases}
$$

Since the domain $D_{\varrho}(A)$ is not dense in $C\left(\bar{B}_{\varrho}\right)$, strong continuity at 0 fails: in fact, $T_{\varrho}(t) f$ converges uniformly to $f$ in $\bar{B}_{\varrho}$ as $t \rightarrow 0$ (that is, $u_{\varrho}$ is continuous up to $t=0)$ if and only if $f \in C_{0}\left(B_{\varrho}\right)$. However, $T_{\varrho}(t) f$ converges to $f$ uniformly in $\bar{B}_{\sigma}$ as $t \rightarrow 0$, for every $\sigma<\varrho$, hence pointwise in $B_{\varrho}$.

An argument based on the classical maximum principle shows that for every $f \in C_{\mathrm{b}}\left(\mathbb{R}^{N}\right)$ the $\operatorname{limit}_{\lim _{\varrho \rightarrow \infty}} T_{\varrho}(t) f$ exists uniformly on compact sets in $\mathbb{R}^{N}$ and defines a semigroup $(T(t))_{t \geq 0}$ of positive contractions in $C_{\mathrm{b}}\left(\mathbb{R}^{N}\right)$. The main properties of $(T(t))_{t \geq 0}$ are listed in the following proposition.

Proposition 2.1. The semigroup $(T(t))_{t \geq 0}$ is irreducible and has the strong Feller property. It is represented by an integral kernel $p$, that is,

$$
T(t) f(x)=\int_{\mathbb{R}^{N}} p(t, x, y) f(y) d y, \quad f \in C_{\mathrm{b}}\left(\mathbb{R}^{N}\right),
$$

where for every fixed $x, t, p(t, x, \cdot)$ is a positive $L^{1}$-function. Moreover, if we set $u(t, x)=T(t) f(x)$ for $t \geq 0, x \in \mathbb{R}^{N}$, then $u$ belongs to the space $C_{\mathrm{loc}}^{1+\alpha / 2,2+\alpha}(] 0, \infty\left[\times \mathbb{R}^{N}\right) \cap C\left(\left[0, \infty\left[\times \mathbb{R}^{N}\right)\right.\right.$ and solves $(1.2)$.

We recall that $(T(t))_{t \geq 0}$ is irreducible if $T(t) f(x)>0$ for every $t>0$, $x \in \mathbb{R}^{N}$ whenever $f \geq 0, f \not \equiv 0$. Moreover, $(T(t))_{t \geq 0}$ has the strong Feller property if for every bounded Borel function $f$ one has $T(t) f \in C_{\mathrm{b}}\left(\mathbb{R}^{N}\right)$ for $t>0$, where $T(t) f$ is defined as in (2.1). For the sake of brevity, we shall write $p(t, x, B)$ instead of $\int_{B} p(t, x, y) d y$ for Borel sets $B \subset \mathbb{R}^{N}$. 
If $f \geq 0$, then $u(t, x)=T(t) f(x)$ is minimal among the positive solutions of problem (1.2). For this reason, in [2], the semigroup $(T(t))_{t \geq 0}$ is called the minimal semigroup generated by $A$. With the semigroup $(T(t))_{t \geq 0}$ can be associated a unique (up to equivalence) Markov process with continuous paths in $\mathbb{R}^{N}$ having the family $(p(t, x, \cdot))$ of measures as transition probabilities. This process is conservative, that is, no explosion occurs, if and only if $T(t) 1=p\left(t, x, \mathbb{R}^{N}\right)=1$. In this case $u(t, x)=T(t) f(x)$ yields, for every $f \in C_{\mathrm{b}}\left(\mathbb{R}^{N}\right)$, the unique bounded solution of (1.2). When explosion may occur, our construction of $(T(t))_{t \geq 0}$ corresponds to a Markov process with absorbing barrier at infinity, that is, the particle is absorbed at the explosion time. We refer to [2] for a proof of these properties of the underlying process; see in particular [2, Theorem 1.6] and [2, Remark 1.14], where the continuity of paths and the existence of left limits at the explosion time are proved.

The generator of $(T(t))_{t \geq 0}$ can be defined through the Laplace transform of the semigroup, following the approach of [4]. For $\lambda>0, f \in C_{\mathrm{b}}\left(\mathbb{R}^{N}\right)$, we define bounded operators on $C_{\mathrm{b}}\left(\mathbb{R}^{N}\right)$ by the formula

$$
R(\lambda) f(x)=\int_{0}^{\infty} e^{-\lambda t} T(t) f(x) d t, \quad x \in \mathbb{R}^{N} .
$$

The family $(R(\lambda))_{\lambda>0}$ satisfies the resolvent identity and every operator $R(\lambda)$ is injective, so that there exists a (unique) operator $(\widehat{A}, \widehat{D})$ such that $R(\lambda)=$ $R(\lambda, \widehat{A})$. We call the operator $\widehat{A}$ the generator of $(T(t))_{t \geq 0}$, in analogy with the terminology used for strongly continuous semigroups.

The above approach leads to the definition of the resolvent of the generator rather than to that of the generator itself. In some cases it is more practical to use the following direct description of $(\widehat{A}, \widehat{D})$, whose proof can be found in [25]:

$$
\begin{aligned}
& \widehat{D}=\left\{u \in C_{\mathrm{b}}\left(\mathbb{R}^{N}\right): \sup _{t>0}\left\|\frac{T(t) u-u}{t}\right\|_{\infty}<\infty, \exists g \in C_{\mathrm{b}}\left(\mathbb{R}^{N}\right)\right. \\
& \text { such that } \left.\lim _{t \rightarrow 0} \frac{T(t) u(x)-u(x)}{t}=g(x) \forall x \in \mathbb{R}^{N}\right\}, \\
& \widehat{A} u(x)=\lim _{t \rightarrow 0} \frac{T(t) u(x)-u(x)}{t} \quad \text { for } u \in \widehat{D} .
\end{aligned}
$$

Keeping the terminology of [12], we say that a sequence $\left(f_{n}\right) \subset C_{\mathrm{b}}\left(\mathbb{R}^{N}\right)$ converges boundedly pointwise to $f \in C_{\mathrm{b}}\left(\mathbb{R}^{N}\right)$ if $\sup _{n}\left\|f_{n}\right\|_{\infty}<\infty$ and $f_{n}(x) \rightarrow f(x)$ as $n \rightarrow \infty$ for every $x \in \mathbb{R}^{N}$. It turns out that the operator $(\widehat{A}, \widehat{D})$ is closed with respect to this kind of convergence (see [25]).

Given $f \in C_{\mathrm{b}}\left(\mathbb{R}^{N}\right)$, the function $u(t, x)=T(t) f(x)$ is a classical solution of (1.2), by construction of the semigroup $(T(t))_{t \geq 0}$. On the other hand, a 
stronger property holds if $f \in \widehat{D}$. In this case $T(t) f \in \widehat{D}$ for every $t \geq 0$ and the equality

$$
D_{t} T(t) f(x)=A T(t) f(x)=T(t) A f(x), \quad t>0, x \in \mathbb{R}^{N},
$$

holds (see [25, Proposition 3.2]). To the function $u$ we may apply the interior Schauder estimates for parabolic equations in the following form (see $[15$, Chapter 3, Section 2]). Fix positive numbers $\varepsilon, \tau, \sigma, \delta$ with $0<\delta<\varepsilon<\tau$. Then there is a constant $C$ such that for every classical solution $v$ of $(1.2)$ we have the inequality

$$
\|v\|_{C^{1+\alpha / 2,2+\alpha}\left([\varepsilon, \tau] \times \bar{B}_{\sigma}\right)} \leq C\|v\|_{C\left([\varepsilon-\delta, \tau+\delta] \times \bar{B}_{\sigma+\delta}\right)} .
$$

Let us now clarify the connections between $A$ and $\widehat{A}$. It turns out that $\widehat{D} \subset D_{\max }(A)$ and that $\widehat{A} u=A u$ for every $u \in \widehat{D}$. For this reason, henceforth we write $A, R(\lambda, A)$ instead of $\widehat{A}, R(\lambda, \widehat{A})$. On the other hand, the identification of $\widehat{D}$ is more difficult and can be done explicitly only in some cases. For example, the equality $\widehat{D}=D_{\max }(A)$ holds if and only if $\lambda-A$ is injective on $D_{\max }(A)$ for some (and hence every) positive $\lambda$, whereas $\widehat{D}=D(A)$ if and only if $R(\lambda, A)$ maps $C_{\mathrm{b}}\left(\mathbb{R}^{N}\right)$ into $C_{0}\left(\mathbb{R}^{N}\right)$.

Clearly $(T(t))_{t \geq 0}$ is not strongly continuous in $C_{\mathrm{b}}\left(\mathbb{R}^{N}\right)$. The maximal subspace of strong continuity is given by

$$
Z=\left\{f \in C_{\mathrm{b}}\left(\mathbb{R}^{N}\right):\|T(t) f-f\|_{\infty} \rightarrow 0 \text { as } t \rightarrow 0\right\} .
$$

$Z$ is $T(t)$-invariant, contains $C_{0}\left(\mathbb{R}^{N}\right)$ and coincides with the closure of $\widehat{D}$ in $C_{\mathrm{b}}\left(\mathbb{R}^{N}\right)$.

2.2. Radial operators. Sometimes we shall use comparison arguments with radial operators, i.e., operators $A$ such that $A u$ depends only upon $|x|$ if $u$ depends only upon $|x|$. This technique is classical and goes back to Has'minskiı̌ (see [17], [18]). It is presented e.g. in [26, Sec. 10.2].

Let us now recall some definitions and results from Feller's theory of one-dimensional diffusion processes.

We consider a general second order ordinary differential operator $A y=$ $a\left(y^{\prime \prime}+b y^{\prime}\right)$ on a (possibly unbounded) open interval $\left.I:=\right] r_{1}, r_{2}[a, b$ continuous functions in $I, a$ positive, and the associated abstract Cauchy problem in $C(\bar{I})$

$$
\left\{\begin{array}{l}
D_{t} y(t)=A y(t), \quad t>0 \\
y(0)=f
\end{array}\right.
$$

where $f \in C(\bar{I})$. Using the Wronskian

$$
W(r):=\exp \left\{-\int_{r_{0}}^{r} b(s) d s\right\},
$$


with a fixed $r_{0} \in I$, we define the following functions:

$$
Q(r):=\frac{1}{a(r) W(r)} \int_{r_{0}}^{r} W(s) d s, \quad R(r):=W(r) \int_{r_{0}}^{r} \frac{1}{a(s) W(s)} d s,
$$

and recall Feller's classification of the endpoints $r_{1}, r_{2}$, according to the boundary behaviour of $Q$ and $R$. The endpoint $r_{2}$ is said to be

regular if $Q \in L^{1}(] r_{0}, r_{2}[), R \in L^{1}(] r_{0}, r_{2}[)$,

an exit boundary if $Q \notin L^{1}(] r_{0}, r_{2}[), R \in L^{1}(] r_{0}, r_{2}[)$,

an entrance boundary if $Q \in L^{1}(] r_{0}, r_{2}[), R \notin L^{1}(] r_{0}, r_{2}[)$,

natural if $Q \notin L^{1}(] r_{0}, r_{2}[), R \notin L^{1}(] r_{0}, r_{2}[)$.

Of course, analogous definitions are understood for $r_{1}$. If $r_{2}$ is regular or an exit boundary, then it is called accessible, because, in terms of Markov processes, there is a positive probability that the particle reaches the endpoint $r_{2}$ in a finite time. Otherwise, $r_{2}$ is inaccessible (see [14]). Observe that $r_{2}$ is accessible if and only if $R \in L^{1}(] r_{0}, r_{2}[)$. In [11, Section VI.4.c] the relation between the type of endpoint and the boundary behaviour of the solutions of

$$
\lambda y-\left[a\left(y^{\prime \prime}+b y^{\prime}\right)\right]=0
$$

is discussed in a comprehensive way. Here we state a summary of the results, due to W. Feller ([13]).

THEOREM 2.2. (i) The boundary point $r_{2}$ is regular if and only if there exist two positive decreasing solutions $y_{1}$ and $y_{2}$ of (2.10) satisfying

$$
\lim _{r \rightarrow r_{2}} y_{1}(r)=0, \quad \lim _{r \rightarrow r_{2}} \frac{y_{1}^{\prime}(r)}{W(r)}=-1, \quad \lim _{r \rightarrow r_{2}} y_{2}(r)=1, \quad \lim _{r \rightarrow r_{2}} \frac{y_{2}^{\prime}(r)}{W(r)}=0 .
$$

(ii) The boundary point $r_{2}$ is an exit boundary if and only if every solution of (2.10) is bounded at $r_{2}$ and every positive decreasing solution $y_{1}$ satisfies

$$
\lim _{r \rightarrow r_{2}} y_{1}(r)=0, \quad \lim _{r \rightarrow r_{2}} \frac{y_{1}^{\prime}(r)}{W(r)} \leq 0 .
$$

(iii) The boundary point $r_{2}$ is an entrance boundary if and only if there exists a positive decreasing solution $y_{1}$ of (2.10) satisfying

$$
\lim _{r \rightarrow r_{2}} y_{1}(r)=1, \quad \lim _{r \rightarrow r_{2}} \frac{y_{1}^{\prime}(r)}{W(r)}=0
$$

and every solution of (2.10) independent of $y_{1}$ is unbounded at $r_{2}$. In this case, no nonzero solution tends to 0 as $r$ tends to $r_{2}$. 
(iv) The boundary point $r_{2}$ is natural if and only if there exists a positive, decreasing solution $y_{1}$ of (2.10) satisfying

$$
\lim _{r \rightarrow r_{2}} y_{1}(r)=0, \quad \lim _{r \rightarrow r_{2}} \frac{y_{1}^{\prime}(r)}{W(r)}=0
$$

and every solution of (2.10) independent of $y_{1}$ is unbounded at $r_{2}$.

In cases (i) and (ii), all solutions of (2.10) are bounded near $r_{2}$ and there is a positive, increasing solution $z$ such that $\lim _{r \rightarrow r_{2}} z(r)=1$. In cases (iii) and (iv), every positive increasing solution $z$ satisfies $\lim _{r \rightarrow r_{2}} z(r)=\infty$.

The following lemma (see [22, Lemma 2.2]) allows us to compare the boundaries of different operators when the coefficients satisfy suitable inequalities.

LEMMA 2.3. Suppose that the coefficients $b_{1}, b_{2}$ of the operators $A_{1} y=$ $a_{1}\left(y^{\prime \prime}+b_{1} y^{\prime}\right), A_{2} y=a_{2}\left(y^{\prime \prime}+b_{2} y^{\prime}\right)$ satisfy the inequality $b_{1} \leq b_{2}$ in I. Let $R_{1}, Q_{1}, R_{2}, Q_{2}$ be the functions defined in (2.9) relative to $A_{1}, A_{2}$, respectively. If $a_{1} \leq a_{2}$ in $I$, then $R_{2}(r) \leq R_{1}(r)$ for $r \geq r_{0}$, whereas if $a_{1} \geq a_{2}$ in $I$, then $Q_{1}(r) \leq Q_{2}(r)$ for $r \geq r_{0}$.

We exploit the above information on the one-dimensional case in the study of the $N$-dimensional situation. Let us introduce polar coordinates in $\mathbb{R}^{N}, r \geq 0$ and $\omega \in \partial B_{1}$, so that $|x|=r$ and $x=r \omega$. For radial functions $u(x)=y(r)$, we may write $A u$ in a more convenient way. Observe that for such a function $u$,

$$
u_{x_{i}}=\frac{x_{i}}{r} y^{\prime}(r), \quad u_{x_{i} x_{j}}=\frac{x_{i} x_{j}}{r^{2}} y^{\prime \prime}(r)+\left(\delta_{i j}-\frac{x_{i} x_{j}}{r^{2}}\right) \frac{y^{\prime}(r)}{r},
$$

whence

$$
\begin{aligned}
A u(x)= & \frac{1}{r^{2}} \sum_{i, j=1}^{N} a_{i j}(x) x_{i} x_{j} y^{\prime \prime}(r) \\
& +\frac{1}{r}\left(\sum_{i=1}^{N} a_{i i}(x)-\frac{1}{r^{2}} \sum_{i, j=1}^{N} a_{i j}(x) x_{i} x_{j}+\sum_{i=1}^{N} b_{i}(x) x_{i}\right) y^{\prime}(r) .
\end{aligned}
$$

To simplify the expression, we set, keeping the notation of [23],

$$
\mathcal{Q}(x):=\frac{1}{r^{2}} \sum_{i, j=1}^{N} a_{i j}(x) x_{i} x_{j}, \quad \mathcal{A}(x):=\frac{1}{\mathcal{Q}(x)} \sum_{i=1}^{N}\left(a_{i i}(x)+b_{i}(x) x_{i}\right)
$$

so that we may write

$$
A u(x)=\mathcal{Q}(x)\left[y^{\prime \prime}(r)+\frac{\mathcal{A}(x)-1}{r} y^{\prime}(r)\right] .
$$


Define the maximal and minimal radial symmetrisations of $\mathcal{Q}$ and $\mathcal{A}$ as

$$
\begin{aligned}
\mathcal{Q}^{+}(r) & :=\max _{|x|=r} \mathcal{Q}(x), & \mathcal{Q}^{-}(r):=\min _{|x|=r} \mathcal{Q}(x), \\
\mathcal{A}^{+}(r) & :=\max _{|x|=r} \mathcal{A}(x), & \mathcal{A}^{-}(r):=\min _{|x|=r} \mathcal{A}(x) .
\end{aligned}
$$

Putting them in place of $\mathcal{Q}, \mathcal{A}$ in (2.11), we may introduce various ordinary differential operators on $(0, \infty)$ that can be used for comparison with $A$, thinking of the real variable as the radial variable $r=|x|$ and of the unknown as a function on $\mathbb{R}^{N}$ independent of $\omega$. Set

$$
\begin{aligned}
& B^{+} y(r)=\mathcal{Q}^{+}(r)\left[y^{\prime \prime}(r)+r^{-1}\left(\mathcal{A}^{+}(r)-1\right) y^{\prime}(r)\right], \\
& B^{-} y(r)=\mathcal{Q}^{-}(r)\left[y^{\prime \prime}(r)+r^{-1}\left(\mathcal{A}^{-}(r)-1\right) y^{\prime}(r)\right], \\
& C^{+} y(r)=\mathcal{Q}^{+}(r)\left[y^{\prime \prime}(r)+r^{-1}\left(\mathcal{A}^{-}(r)-1\right) y^{\prime}(r)\right] \\
& C^{-} y(r)=\mathcal{Q}^{-}(r)\left[y^{\prime \prime}(r)+r^{-1}\left(\mathcal{A}^{+}(r)-1\right) y^{\prime}(r)\right] .
\end{aligned}
$$

In what follows, with a slight abuse which should not create any confusion, we feel free to think of the above operators as ordinary differential operators on the half-line or as radial operators in $\mathbb{R}^{N}$, when dealing with functions depending only upon $|x|$.

In the following proposition, whose proof can be found in [22, Proposition 2.3 , we compare the boundary $\infty$ for the operators above.

Proposition 2.4. (i) If $\infty$ is accessible for $B^{-}$then it is accessible for $C^{-}, C^{+}$and $B^{+}$. Similarly, if $\infty$ is accessible for $C^{-}$or for $C^{+}$, then it is accessible for $B^{+}$as well.

(ii) If $\infty$ is regular for $B^{-}$or $C^{-}$then it is regular for $C^{+}$or $B^{+}$, respectively. Conversely, if $\infty$ is natural for $C^{+}$or $B^{+}$then it is natural for $B^{-}$or $C^{-}$, respectively.

(iii) If $\infty$ is an entrance boundary for $C^{-}$then it is an entrance boundary for $C^{+}, B^{-}$and $B^{+}$. Similarly, if $\infty$ is an entrance boundary for $B^{-}$or $B^{+}$, then it is an entrance boundary for $C^{+}$.

\section{Compactness of the semigroup}

3.1. The case where $(T(t))_{t \geq 0}$ is generated by $(A, D(A))$. In this section we show the compactness of $(T(t))_{t \geq 0}$ when $(A, D(A))$ is its generator. As we have already observed in the preceding section, this is the case if and only if $R(\lambda, A)$ maps $C_{\mathrm{b}}\left(\mathbb{R}^{N}\right)$ into $C_{0}\left(\mathbb{R}^{N}\right)$.

We recall the following result from [22, Section 3$]$; see also [10, Theorems $6.4,6.5]$ and [17], [18].

Proposition 3.1. Suppose that there exists a positive function $V \in$ $C^{2}\left(\mathbb{R}^{N} \backslash \bar{B}_{\varrho}\right)$, for some $\varrho>0$, such that $V(x) \rightarrow 0$ as $|x| \rightarrow \infty$ and $V-A V \geq 1$. Then the generator of $(T(t))_{t \geq 0}$ is $(A, D(A))$. In particular 
this happens if

$$
\lim _{|x| \rightarrow \infty} \frac{\sum_{i=1}^{N}\left(a_{i i}(x)+b_{i}(x) x_{i}\right)-\left[2+(\alpha+1)(\log |x|)^{-1}\right] \mathcal{Q}(x)}{|x|^{2}(\log |x|)^{\alpha+1}}=\infty
$$

for some $\alpha>0$ or if $\infty$ is accessible for $B^{-}$.

An easy example of application of the preceding result is given by the operator $A u=\Delta u+c r^{\gamma} u_{r}$; in this case, (3.1) holds if and only if $c>0$ and $\gamma>1$.

Part (i) of the following result is proved in [10, Theorem 6.1] if $A$ is self-adjoint.

Theorem 3.2. If $(A, D(A))$ is the generator of $(T(t))_{t \geq 0}$, then the following statements hold:

(i) $R(\lambda, A)$ is a compact operator.

(ii) For every $t>0, T(t)$ maps $C_{\mathrm{b}}\left(\mathbb{R}^{N}\right)$ into $C_{0}\left(\mathbb{R}^{N}\right)$ and is a compact operator.

(iii) $\|T(t)\| \rightarrow 0$ exponentially as $t \rightarrow \infty$.

(iv) The semigroup is norm-continuous for $t>0$, that is,

$$
\lim _{h \rightarrow 0}\|T(t+h)-T(t)\|=0 \quad \text { for every } t>0 .
$$

Proof. (i) Let $\mathcal{U}$ be the unit ball of $C_{\mathrm{b}}\left(\mathbb{R}^{N}\right)$. Set $u_{f}:=R(\lambda, A) f$ and $v=R(\lambda, A) 1$. The positivity of $R(\lambda, A)$ yields $\left|u_{f}\right| \leq v$ for all $f \in \mathcal{U}$. Fix $\varepsilon>0$; by hypothesis, there is $\sigma>0$ such that $\left|u_{f}(x)\right| \leq v(x) \leq \varepsilon$ for $|x| \geq \sigma$ for all $f \in \mathcal{U}$. From the inequality

$$
\left\|u_{f}\right\|_{W^{2, p}\left(B_{\sigma}\right)} \leq c\left[\|f\|_{L^{p}\left(B_{\varrho}\right)}+\left\|u_{f}\right\|_{L^{p}\left(B_{\varrho}\right)}\right] \leq c_{1} \quad \forall f \in \mathcal{U},
$$

with $p>N, \sigma<\varrho$, it follows that the norms of $u_{f}$ in $C^{1}\left(\bar{B}_{\sigma}\right)$ are uniformly bounded. Then, by Ascoli's theorem, the family $\left\{\left.u_{f}\right|_{\bar{B}_{\sigma}}: f \in \mathcal{U}\right\}$ is totally bounded in $C\left(\bar{B}_{\sigma}\right)$. From these facts it is easy to see that the family $\left\{u_{f}\right.$ : $f \in \mathcal{U}\}$ can be covered by a finite number of $2 \varepsilon$-balls of $C_{0}\left(\mathbb{R}^{N}\right)$. This yields the compactness of $R(\lambda, A)$.

(ii) First we show that $w(t, x):=T(t) 1(x)$ belongs to $C_{0}\left(\mathbb{R}^{N}\right)$ for every fixed $t>0$. Observe that $0 \leq w(t+\tau, x) \leq w(t, x)$ for all $t, \tau \geq 0, x \in \mathbb{R}^{N}$. In fact

$$
w(t+\tau, x)=\int_{\mathbb{R}^{N}} p(t, x, y) w(\tau, y) d y \leq \int_{\mathbb{R}^{N}} p(t, x, y) d y=w(t, x) .
$$

Recall moreover that

$$
R(\lambda, A) 1(x)=\int_{0}^{\infty} e^{-\lambda t} w(t, x) d t
$$


belongs to $C_{0}\left(\mathbb{R}^{N}\right)$, i.e.,

$$
\lim _{|x| \rightarrow \infty} \int_{0}^{\infty} e^{-\lambda t} w(t, x) d t=0 .
$$

Let $\left(x_{n}\right) \subset \mathbb{R}^{N}$ be such that $\left|x_{n}\right| \rightarrow \infty$ as $n \rightarrow \infty$. By the Fatou lemma

$$
\int_{0}^{\infty} e^{-\lambda t} \liminf _{n \rightarrow \infty} w\left(t, x_{n}\right) d t \leq \liminf _{n \rightarrow \infty} \int_{0}^{\infty} e^{-\lambda t} w\left(t, x_{n}\right) d t=0
$$

whence $\liminf \inf _{n \rightarrow \infty} w\left(t, x_{n}\right)=0$ for almost every $t \geq 0$. Choosing $t_{1}>0$ such that $\liminf _{n \rightarrow \infty} w\left(t_{1}, x_{n}\right)=0$, since $0 \leq w\left(t_{1}+\tau, x_{n}\right) \leq w\left(t_{1}, x_{n}\right)$ for every $\tau>0$, we deduce that $\liminf _{n \rightarrow \infty} w\left(t, x_{n}\right)=0$ for all $t \geq t_{1}$. Since $t_{1}$ can be chosen as close to 0 as we want, it follows that $\liminf _{n \rightarrow \infty} w\left(t, x_{n}\right)=0$ for all $t>0$. By the arbitrariness of the sequence $\left(x_{n}\right)$, we conclude that $w(t, \cdot)$ lies in $C_{0}\left(\mathbb{R}^{N}\right)$.

At this point the proof goes along the same lines as for (i). Keeping the above notation we fix $\varepsilon>0$ and obtain $|T(t) f(x)| \leq T(t) 1(x) \leq \varepsilon$ for all $f \in \mathcal{U}$ and $|x| \geq \varrho$ for a suitable $\varrho>0$. The interior Schauder estimates (2.5) imply that

$$
\|T(t) f\|_{C^{1}\left(\bar{B}_{\varrho}\right)} \leq C
$$

for every $f \in \mathcal{U}$ so that, by Ascoli's theorem and the above estimate, one concludes that $T(t) \mathcal{U}$ can be covered by a finite number of $2 \varepsilon$-balls in $C_{0}\left(\mathbb{R}^{N}\right)$.

(iii) Since $T(t) 1$ belongs to $C_{0}\left(\mathbb{R}^{N}\right)$ and $T(t) 1(x)<1$ for every $x \in \mathbb{R}^{N}$, from the parabolic strong maximum principle (see [15]) we deduce that $\|T(t)\|=\|T(t) 1\|_{\infty}<1$ for $t>0$. The semigroup law now yields the exponential decay.

(iv) Since $T(t)$ maps $C_{\mathrm{b}}\left(\mathbb{R}^{N}\right)$ into $C_{0}\left(\mathbb{R}^{N}\right)$, and $C_{0}\left(\mathbb{R}^{N}\right) \subset Z$, where $Z$ is the maximal subspace of strong continuity defined in (2.6), we deduce that

$$
\lim _{h \rightarrow 0}\|(T(h)-I) T(t) f\|_{\infty}=0
$$

for every $t>0$ and $f \in C_{\mathrm{b}}\left(\mathbb{R}^{N}\right)$. Since $T(t)$ is a compact operator, equality (3.2) holds uniformly on the unit ball of $C_{\mathrm{b}}\left(\mathbb{R}^{N}\right)$ and the statement is proved.

Corollary 3.3. If $T(t)$ maps $C_{\mathrm{b}}\left(\mathbb{R}^{N}\right)$ into $C_{0}\left(\mathbb{R}^{N}\right)$ for $t>0$ then $(A, D(A))$ is the generator of $(T(t))_{t \geq 0}$ and therefore Theorem 3.2 applies.

Proof. By the proof of Theorem 3.2(iv), $T(t)$ is norm-continuous for $t>0$. Therefore the integral yielding the representation

$$
R(\lambda, A)=\int_{0}^{\infty} e^{-\lambda t} T(t) d t
$$


converges in the operator norm of $C_{\mathrm{b}}\left(\mathbb{R}^{N}\right)$ and shows that $R(\lambda, A)$ maps $C_{\mathrm{b}}\left(\mathbb{R}^{N}\right)$ into $C_{0}\left(\mathbb{R}^{N}\right)$, hence the generator of $(T(t))_{t \geq 0}$ is $(A, D(A))$.

A partial converse to the above theorem is stated in the following proposition.

Proposition 3.4. If $R(\lambda, A)$ preserves $C_{0}\left(\mathbb{R}^{N}\right)$ and is compact as an operator from $C_{0}\left(\mathbb{R}^{N}\right)$ into itself, then $R(\lambda, A)$ maps $C_{\mathrm{b}}\left(\mathbb{R}^{N}\right)$ into $C_{0}\left(\mathbb{R}^{N}\right)$, the generator of $(T(t))_{t \geq 0}$ is $(A, D(A))$ and $\infty$ is accessible for $B^{+}$.

Proof. Given $f \in C_{\mathrm{b}}\left(\mathbb{R}^{N}\right)$, let $\left(f_{n}\right)$ be a bounded sequence in $C_{0}\left(\mathbb{R}^{N}\right)$ such that $f_{n} \rightarrow f$ uniformly on compact subsets of $\mathbb{R}^{N}$. Since $\left(f_{n}\right)$ is bounded in $C_{0}\left(\mathbb{R}^{N}\right)$, by hypothesis, possibly extracting a subsequence, the sequence $\left(R(\lambda, A) f_{n}\right)$ converges to a function $u \in C_{0}\left(\mathbb{R}^{N}\right)$. The interior $L^{p}$ estimates (see [16, Theorem 9.11] and the proof of Theorem 3.4 in [22]) give $u \in D_{0}(A)$ and $\lambda u-A u=f$. Since $D_{0}(A) \subset \widehat{D}$ (see [22, Proposition 3.5]), we obtain $R(\lambda, A) f=u \in C_{0}\left(\mathbb{R}^{N}\right)$. The last statement follows from Corollary 3.15 in $[22]$.

3.2. The case where $(T(t))_{t \geq 0}$ is generated by $\left(A, D_{\max }(A)\right)$. In this subsection we assume that $(T(t))_{t \geq 0}$ is generated by $\left(A, D_{\max }(A)\right)$. We give two sufficient conditions, the first (Theorem 3.10) based upon the existence of a suitable Lyapunov function, the second (Theorem 3.12) relying upon comparison with a radial operator. We recall that the generator of $(T(t))_{t \geq 0}$ is $\left(A, D_{\max }(A)\right)$ if and only if $\lambda-A$ is injective on $D_{\max }(A)$ or, in an equivalent way, if $T(t) 1=1$ for every $t \geq 0$. A sufficient condition is the existence of a function $V \in C^{2}\left(\mathbb{R}^{N} \backslash \bar{B}_{\varrho}\right)$, for some $\varrho>0$, such that $V(x) \rightarrow \infty$ as $|x| \rightarrow \infty$, and $\lambda V-A V \geq 0$ for some positive $\lambda$. The existence of such a function is in turn ensured by various conditions. Some of them are collected in the following proposition, whose proof can be found in [22] (see also [10] and [17], [18]).

Proposition 3.5. Suppose that

$$
\sum_{i=1}^{N}\left(a_{i i}(x)+b_{i}(x) x_{i}\right)-2 \mathcal{Q}(x) \leq C|x|^{2} \log |x|
$$

for some $C>0$ and $x$ large, or that $\infty$ is inaccessible for $B^{+}$. Then $\lambda-A$ is injective on $D_{\max }(A)$.

Consider again the operator $A u=\Delta u+c r^{\gamma} u_{r}$; in this case, (3.3) holds if and only if $c \leq 0$ or $c>0$ and $\gamma \leq 1$.

The following proposition is the basic tool for our investigation of compactness when $(T(t))_{t \geq 0}$ is generated by $\left(A, D_{\max }(A)\right)$. 
Proposition 3.6. Suppose that $\lambda-A$ is injective on $D_{\max }(A)$. Then the semigroup $(T(t))_{t \geq 0}$ is compact in $C_{\mathrm{b}}\left(\mathbb{R}^{N}\right)$ if and only if for every $t, \varepsilon>0$ there exists a radius $\varrho=\varrho(t, \varepsilon)$ such that

$$
p\left(t, x, B_{\varrho}\right) \geq 1-\varepsilon
$$

for all $x \in \mathbb{R}^{N}$, where $p$ is the integral kernel of $(T(t))_{t \geq 0}$ given by Proposition 2.1.

Proof. Suppose that the operators $T(t)$ are compact for all $t>0$. Consider functions $f_{\varrho} \in C_{0}\left(\mathbb{R}^{N}\right)$ satisfying $\chi_{B_{\varrho-1}} \leq f_{\varrho} \leq \chi_{B_{\varrho}}$ for $\varrho>1$. Since $f_{\varrho} \rightarrow 1$ uniformly on compact sets and $T(t) 1=1$, it follows that $\lim _{\varrho \rightarrow \infty}\left\|T(t) f_{\varrho}-1\right\|_{\infty}=0$. Since

$$
p\left(t, x, B_{\varrho}\right) \geq T(t) f_{\varrho}(x),
$$

for any $\varepsilon>0$ and $t>0$ there is $\varrho>0$ such that (3.4) holds.

Fix now $\varepsilon>0$ and $t>0$, and write $t=\tau+h$ with $\tau, h>0$. Let $\varrho>0$ be such that (3.4) holds with $\tau$ instead of $t$. We have

$$
T(t) f(x)=T(\tau+h) f(x)=\int_{\mathbb{R}^{N}} p(\tau, x, y) T(h) f(y) d y
$$

for every $f \in C_{\mathrm{b}}\left(\mathbb{R}^{N}\right)$. Define now

$$
T_{\varepsilon} f(x):=\int_{B_{\varrho}} p(\tau, x, y) T(h) f(y) d y=(S \circ \mathcal{R} \circ T(h) f)(x),
$$

where $S: C\left(\bar{B}_{\varrho}\right) \rightarrow C_{\mathrm{b}}\left(\mathbb{R}^{N}\right)$ is defined by

$$
S f(x)=\int_{B_{\varrho}} p(\tau, x, y) f(y) d y
$$

and $\mathcal{R}$ is the restriction operator $\mathcal{R}: C_{\mathrm{b}}\left(\mathbb{R}^{N}\right) \rightarrow C\left(\bar{B}_{\varrho}\right)$. From the interior Schauder estimates (2.5) we deduce the compactness of the operator $\mathcal{R} \circ T(h)$ (see the proof of Theorem 3.2(ii)) and of $T_{\varepsilon}$. Since $\left\|T(t)-T_{\varepsilon}\right\| \leq \varepsilon, T(t)$ is compact.

Remark 3.7. Let $\lambda-A$ be injective on $D_{\max }(A)$ and fix $\varepsilon, \varrho>0$. If (3.4) holds for some $\bar{t}>0$ and every $x \in \mathbb{R}^{N}$ then it holds for every $t>\bar{t}$ and $x \in \mathbb{R}^{N}$. In fact, writing $t=\bar{t}+\tau$, with $\tau>0$, we have

$$
p\left(t, x, B_{\varrho}\right)=\int_{\mathbb{R}^{N}} p(\tau, x, y) p\left(\bar{t}, y, B_{\varrho}\right) d y \geq(1-\varepsilon) \int_{\mathbb{R}^{N}} p(\tau, x, y) d y=1-\varepsilon .
$$

In order to prove our first compactness condition we need some preliminary lemmas.

Let $V \in C^{2}\left(\mathbb{R}^{N}\right)$ be a positive function such that $\lim _{|x| \rightarrow \infty} V(x)=\infty$; for $\alpha \geq 0$, set $V_{\alpha}=V \wedge \alpha$ and $u_{\alpha}(t, x)=T(t) V_{\alpha}(x)$. 
LEMMA 3.8. With the notation above, the inequality

$$
D_{t} u_{\alpha}(t, x) \leq \int_{\{V \leq \alpha\}} p(t, x, y) A V(y) d y
$$

holds for every $t \geq 0$ and $x \in \mathbb{R}^{N}$.

Proof. For every $\varepsilon>0$ let $\psi_{\varepsilon} \in C^{\infty}(\mathbb{R})$ be such that $\psi_{\varepsilon}(t)=t$ for $t \leq \alpha$, $\psi_{\varepsilon}$ constant in $\left[\alpha+\varepsilon, \infty\left[, \psi_{\varepsilon}^{\prime} \geq 0, \psi_{\varepsilon}^{\prime \prime} \leq 0\right.\right.$. Observe that $\psi_{\varepsilon}(t) \rightarrow t \wedge \alpha$ and $\psi_{\varepsilon}^{\prime}(t) \rightarrow \chi_{]-\infty, \alpha]}(t)$ pointwise as $\varepsilon \rightarrow 0$. Since the function $\psi_{\varepsilon} \circ V$ belongs to $D_{\max }(A)$, we have

$$
D_{t} T(t)\left(\psi_{\varepsilon} \circ V\right)(x)=\int_{\mathbb{R}^{N}} p(t, x, y) A\left(\psi_{\varepsilon} \circ V\right)(y) d y .
$$

On the other hand,

$$
\begin{aligned}
A\left(\psi_{\varepsilon} \circ V\right)(x) & =\psi_{\varepsilon}^{\prime}(V(x)) A V(x)+\psi_{\varepsilon}^{\prime \prime}(V(x)) \sum_{i, j=1}^{N} a_{i j}(x) D_{i} V(x) D_{j} V(x) \\
& \leq \psi_{\varepsilon}^{\prime}(V(x)) A V(x)
\end{aligned}
$$

and hence

$$
D_{t} T(t)\left(\psi_{\varepsilon} \circ V\right)(x) \leq \int_{\mathbb{R}^{N}} p(t, x, y) \psi_{\varepsilon}^{\prime}(V(y)) A V(y) d y .
$$

Observe that $\psi_{\varepsilon} \circ V \leq \alpha+1$ and $\psi_{\varepsilon} \circ V \rightarrow V_{\alpha}$ pointwise as $\varepsilon \rightarrow 0$. From Proposition 4.6 in [22] we deduce that $T(t)\left(\psi_{\varepsilon} \circ V\right) \rightarrow u_{\alpha}$ uniformly on compact sets of $] 0, \infty\left[\times \mathbb{R}^{N}\right.$, hence the interior Schauder estimates $(2.5)$ imply that $D_{t} T(t)\left(\psi_{\varepsilon} \circ V\right) \rightarrow D_{t} u_{\alpha}$ pointwise. Letting $\varepsilon \rightarrow 0$ in (3.5) we obtain the assertion by dominated convergence.

Lemma 3.9. Suppose that $A V \leq K V$ for some $K>0$. Then for every $t>0, x \in \mathbb{R}^{N}$ the functions $V$ and $|A V|$ are summable with respect to the measure $p(t, x, \cdot)$. If we set

$$
u(t, x)=\int_{\mathbb{R}^{N}} p(t, x, y) V(y) d y
$$

the function $u$ belongs to $C^{1,2}(] 0, \infty\left[\times \mathbb{R}^{N}\right) \cap C\left(\left[0, \infty\left[\times \mathbb{R}^{N}\right)\right.\right.$ and satisfies the inequalities

$$
u(t, x) \leq e^{K t} V(x), \quad D_{t} u(t, x) \leq \int_{\mathbb{R}^{N}} p(t, x, y) A V(y) d y .
$$

Proof. From Lemma 3.8 we obtain

$$
D_{t} u_{\alpha}(t, x) \leq \int_{\{V \leq \alpha\}} p(t, x, y) A V(y) d y \leq K u_{\alpha}(t, x)
$$

and hence, by Gronwall's lemma, $u_{\alpha}(t, x) \leq e^{K t} V_{\alpha}(x)$. Letting $\alpha \rightarrow \infty$ we obtain $u(t, x) \leq e^{K t} V(x)$ and then $V$ is summable with respect to the 
measure $p(t, x, \cdot)$. The inequality $0 \leq u_{\alpha} \leq u$ and the interior Schauder estimates (2.5) show that $\left(u_{\alpha}\right)$ is relatively compact in $C^{1,2}(] 0, \infty\left[\times \mathbb{R}^{N}\right)$. Since $u_{\alpha} \rightarrow u$ pointwise as $\alpha \rightarrow \infty$, it follows that $u \in C^{1,2}(] 0, \infty\left[\times \mathbb{R}^{N}\right)$. Moreover, the inequality $u_{\alpha}(t, x) \leq u(t, x) \leq e^{K t} V(x)$ implies that $u(t, \cdot) \rightarrow$ $V(\cdot)$ as $t \rightarrow 0^{+}$, uniformly on compact sets. Set $E=\left\{x \in \mathbb{R}^{N}: A V(x) \geq 0\right\}$. Clearly

$$
\int_{E} p(t, x, y) A V(y) d y \leq K \int_{E} p(t, x, y) V(y) d y \leq K u(t, x)<\infty .
$$

Moreover, letting $\alpha \rightarrow \infty$ in (3.7) we obtain

$$
D_{t} u(t, x) \leq \liminf _{\alpha \rightarrow \infty} \int_{\{V \leq \alpha\}} p(t, x, y) A V(y) d y .
$$

This fact and (3.8) imply that $|A V|$ is summable with respect to $p(t, x, \cdot)$ and that the above liminf is a limit, so that the proof is complete.

We now prove the announced condition for compactness.

THEOREM 3.10. Suppose that there exists $V \in C^{2}\left(\mathbb{R}^{N} \backslash \bar{B}_{\varrho}\right)$, for some $\varrho>0$, such that $\lim _{|x| \rightarrow \infty} V(x)=\infty, A V \leq-g(V)$ where $g:[0, \infty[\rightarrow \mathbb{R}$ is a convex function satisfying $\lim _{x \rightarrow \infty} g(x)=\infty$ and such that $1 / g$ is summable in a neighbourhood of $\infty$. Then the semigroup $(T(t))_{t \geq 0}$ is compact in $C_{\mathrm{b}}\left(\mathbb{R}^{N}\right)$.

Proof. Multiplying $V$ by a smooth function equal to 1 in a neighbourhood of $\infty$ and to 0 in the set $\{V \leq 0\}$ and, possibly, replacing $g$ with $g+c$ for a suitable $c \in \mathbb{R}$, we may assume that $0 \leq V \in C^{2}\left(\mathbb{R}^{N}\right)$.

Clearly $\lambda V-A V \geq 0$ in $\mathbb{R}^{N} \backslash \bar{B}_{\varrho}$ for some positive $\lambda$ and hence $\lambda-A$ is injective on $D_{\max }(A)$. From Lemma 3.9 and Jensen's inequality, for $u$ given by (3.6), we obtain

$$
D_{t} u(t, x) \leq \int_{\mathbb{R}^{N}} p(t, x, y) A V(y) d y \leq-\int_{\mathbb{R}^{N}} p(t, x, y) g(V(y)) d y \leq-g(u(t, x))
$$

and therefore $u(t, x) \leq z(t, x)$, where $z$ is the solution of the ordinary Cauchy problem

$$
\left\{\begin{array}{l}
D_{t} z=-g(z) \\
z(0, x)=V(x)
\end{array}\right.
$$

Since $1 / g$ is summable near $\infty$, it is easily seen that for every $\bar{t}>0$ there is a constant $C(\bar{t})$ such that $z(t, x) \leq C(\bar{t})$ for every $t \geq \bar{t}$ and $x \in \mathbb{R}^{N}$. Let now $\bar{t}>0$ be fixed, and let $\varepsilon, \varrho>0$ be such that $V(x) \geq 1 / \varepsilon$ for $|x| \geq \varrho$. Then for $t \geq \bar{t}$ we have

$$
p\left(t, x, \mathbb{R}^{N} \backslash B_{\varrho}\right) \leq \varepsilon \int_{\mathbb{R}^{N} \backslash B_{\varrho}} p(t, x, y) V(y) d y \leq \varepsilon u(t, x) \leq \varepsilon C(\bar{t})
$$

for every $x \in \mathbb{R}^{N}$ and the conclusion follows from Proposition 3.6. 
The choice of (simple) functions $V$ and $g$ in the above theorem gives explicit conditions on the coefficients.

Corollary 3.11. Suppose that

$$
\sum_{i=1}^{N}\left(a_{i i}(x)+b_{i}(x) x_{i}\right)-2 \mathcal{Q}(x) \leq-\beta|x|^{2}(\log |x|)^{\gamma}
$$

for $|x|$ large and some $\gamma>1, \beta>0$. Then $(T(t))_{t \geq 0}$ is compact.

Proof. It is sufficient to take $V(x)=\log |x|$ (with $|x|$ large) and $g(s)=$ $\beta s^{\gamma}$ in Theorem 3.10.

Choosing $V(x)=|x|^{2}$ instead of $\log |x|$, we may apply Theorem 3.10 if the inequality

$$
\sum_{i=1}^{N}\left(a_{i i}(x)+b_{i}(x) x_{i}\right) \leq-\beta|x|^{\gamma}
$$

holds for $|x|$ large and some $\gamma>2, \beta>0$. In this case $g(s)=\beta s^{\gamma / 2}$. Condition (3.10) has been assumed in [7] and is clearly stronger than (3.9). The main difference between (3.9) and (3.10), however, is not the logarithmic term in (3.9) that allows $\gamma=2$ but rather the presence of $-2 \mathcal{Q}$ which, in some cases, can compensate for the growth of $\sum_{i=1}^{N} a_{i i}(x)$. Such a phenomenon is exhibited in Example 5.5.

If $1 / g$ is not summable near $\infty$, in general the compactness of $(T(t))_{t \geq 0}$ fails. In fact, if $A$ is the one-dimensional Ornstein-Uhlenbeck operator $A u=u^{\prime \prime}-x u^{\prime}$, the function $V(x)=e^{|x|}$ (for $|x|$ large) satisfies $A V(x) \leq$ $-(1 / 2) V(x) \log V(x)$ for large $|x|$, but the resolvent (hence the semigroup) of $A$ is not compact in $C_{\mathrm{b}}(\mathbb{R})$ (see [24], [21]).

We now formulate a compactness result based on comparison with a radial operator.

Theorem 3.12. If $\infty$ is an entrance boundary for $C^{-}$, then the semigroup $(T(t))_{t \geq 0}$ is compact.

Proof. By Proposition 2.4(iii), $\infty$ is inaccessible for $B^{+}$. Proposition 3.5 then shows that $\lambda-A$ is injective on $D_{\max }(A)$ and therefore the generator of $(T(t))_{t \geq 0}$ is $\left(A, D_{\max }(A)\right)$. In order to prove the compactness of $(T(t))_{t \geq 0}$, we fix $\varepsilon, \bar{t}>0$ and show that there exists $\varrho>0$ such that (3.4) holds for $t=\bar{t}$. For clarity reasons we set $\eta=1-\sqrt{1-\varepsilon}$ and divide the proof into several steps.

STEP 1: There exists $\varrho_{0}$ such that the (unique) bounded solution of the problem

$$
\left\{\begin{array}{l}
z(r)-C^{-} z(r)=0, \quad r>\varrho_{0}, \\
z\left(\varrho_{0}\right)=1,
\end{array}\right.
$$

satisfies $z(r) \geq 1-\eta \bar{t} e^{-\bar{t}}$ for $r \geq \varrho_{0}$. 
In fact, let $y$ be the (unique) positive decreasing solution of the equation $y=C^{-} y$ in $] 0, \infty\left[\right.$ such that $\lim _{r \rightarrow \infty} y(r)=1$ (see Theorem 2.2). Then, if $\varrho_{0}$ is large enough, the function $z(r)=y(r) / y\left(\varrho_{0}\right)$ satisfies the requirements.

STEP 2: The operator $C^{-}$, with domain

$$
D_{\varrho_{0}}=\left\{u \in C _ { \mathrm { b } } ^ { 2 } \left(\left[\varrho_{0}, \infty[): u\left(\varrho_{0}\right)=0\right\},\right.\right.
$$

generates a positive contractive semigroup $(S(t))_{t \geq 0}$ in $C_{\mathrm{b}}\left(\left[\varrho_{0}, \infty[)\right.\right.$ (not strongly continuous). Given $f \in C_{\mathrm{b}}\left(\left[\varrho_{0}, \infty[)\right.\right.$, the function $v(t, r)=S(t) f(r)$ belongs to the space $C^{1,2}(] 0, \infty[\times] \varrho_{0}, \infty[) \cap C\left(\left[0, \infty\left[\times\left[\varrho_{0}+\delta, \infty[)\right.\right.\right.\right.$ for every positive $\delta$ and solves the problem

$$
\begin{cases}D_{t} v(t, r)=C^{-} v(t, r), & t>0, r>\varrho_{0}, \\ v(0, r)=f(r), & r \geq \varrho_{0}, \\ v\left(t, \varrho_{0}\right)=0, & t>0 .\end{cases}
$$

The construction of $(S(t))_{t \geq 0}$ and the verification of the above properties can be achieved with the methods outlined in Section 2, that is, by considering the semigroups $\left(S_{\varrho}(t)\right)_{t \geq 0}$ generated by $C^{-}$with Dirichlet boundary conditions in $C\left(\left[\varrho_{0}, \varrho\right]\right)$ and letting $\varrho \rightarrow \infty$.

In fact, one shows that, for positive $f$, the family $\left(S_{\varrho}(t) f\right)$ is increasing with respect to $\varrho$ and uniformly bounded by $\|f\|_{\infty}$, so that $S(t) f(r)$ is defined pointwise as $\lim _{\varrho \rightarrow \infty} S_{\varrho}(t) f(r)$. Using the interior Schauder estimates (2.5) one deduces that $v$ belongs to $C^{1,2}(] 0, \infty[\times] \varrho_{0}, \infty[)$ and solves (3.11). The fact that $v$ belongs to $C\left(\left[0, \infty\left[\times\left[\varrho_{0}+\delta, \infty[)\right.\right.\right.\right.$ for every positive $\delta$ can be proved by arguing as in Theorem 4.5 of [22]. Finally, since $\varrho_{0}$ is accessible for $C^{-}$, one concludes that the generator of $(S(t))_{t \geq 0}$ is $\left(C^{-}, D_{\varrho 0}\right)$ by arguing as in Theorem 3.5(i) of [22].

STEP 3: The function $v(t, r)=S(t) 1(r)$ is nonincreasing with respect to $t$ for every fixed $r$ and nondecreasing with respect to $r$ for every fixed $t$.

To see that $v$ is nonincreasing with respect to $t$ observe that, since $(S(t))_{t \geq 0}$ is positive and contractive, $v(t+\tau, r)=S(t) v(\tau, \cdot)(r) \leq S(t) 1(r)=$ $v(t, r)$. We now prove that $v$ is nondecreasing with respect to $r$. Note that, for fixed $t, C^{-} v(t, \cdot)=g(\cdot)$ with $g=D_{t} v \leq 0$. We write $C^{-}$in the self-adjoint form

$$
C^{-} y=a W\left(y^{\prime} / W\right)^{\prime}
$$

where

$$
a(r)=\mathcal{Q}^{-}(r), \quad W(r)=\exp \left\{-\int_{\varrho_{0}}^{r} \frac{\mathcal{A}^{+}(s)-1}{s} d s\right\} .
$$

It follows that for every $r, s$ with $\varrho_{0}<r<s$ we have the equality

$$
\frac{D_{r} v(t, s)}{W(s)}-\frac{D_{r} v(t, r)}{W(r)}=\int_{r}^{s} \frac{g(\xi)}{a(\xi) W(\xi)} d \xi .
$$


Since $g \leq 0$, the limit $\lim _{s \rightarrow \infty} D_{r} v(t, s) / W(s)=\ell$ exists with $-\infty \leq \ell \leq$ $+\infty$. If $\ell \neq 0$, e.g. if $\ell>0$, then we obtain $D_{r} v(t, s) \geq c W(s)$ for some positive constant $c$ and $s$ large. This is impossible since $v(t, \cdot)$ is bounded and $W$ is not summable near $\infty$, which is an entrance boundary for $C^{-}$. Therefore $\ell=0$ and, letting $s \rightarrow \infty$ in (3.12), we obtain

$$
\frac{D_{r} v(t, r)}{W(r)}=-\int_{r}^{\infty} \frac{g(\xi)}{a(\xi) W(\xi)} d \xi \geq 0
$$

as claimed.

STEP 4: For $v(t, r)=S(t) 1(r)$, the inequality $0 \leq v(\bar{t}, r) \leq \eta$ holds for every $r \geq \varrho_{0}$.

Let

$$
\psi(r)=\int_{0}^{\infty} e^{-t} v(t, r) d t
$$

be the Laplace transform of $v$ at the point 1 . The function $\psi$ belongs to $D_{\varrho_{0}}$ and solves the problem

$$
\left\{\begin{array}{l}
\psi-C^{-} \psi=1 \\
\psi\left(\varrho_{0}\right)=0
\end{array}\right.
$$

Then $\psi=1-z$, where $z$ is the function constructed in Step 1, and hence $\psi(r) \leq \eta \bar{t} e^{-\bar{t}}$ for $r \geq \varrho_{0}$. Let $\delta>\eta e^{-\bar{t}}$. Using the Chebyshev inequality we deduce that the Lebesgue measure of the set

$$
E=\left\{t \geq 0: e^{-t} \lim _{r \rightarrow \infty} v(t, r) \geq \delta\right\}
$$

is less than or equal to $\eta \bar{t} e^{-\bar{t}} / \delta<\bar{t}$. Hence, there exists $t \leq \bar{t}$ such that $t \notin E$, that is, $e^{-t} v(t, r) \leq e^{-t} \lim _{r \rightarrow \infty} v(t, r)<\delta$. Since $v$ is nonincreasing with respect to $t$, and $\delta>\eta e^{-\bar{t}}$ is arbitrary, it follows that $e^{-\bar{t}} v(\bar{t}, r) \leq \eta$ for $r \geq \varrho_{0}$.

STEP 5 (Conclusion). For every $\varrho>1$ consider a nonnegative function $f_{\varrho} \in C_{0}\left(\mathbb{R}^{N}\right)$ such that $\chi_{B_{\varrho-1}} \leq f_{\varrho} \leq \chi_{B_{\varrho}}$. Since $f_{\varrho} \rightarrow 1$ uniformly on compact sets as $\varrho \rightarrow \infty$, Proposition 4.6 of [22] shows the existence of a radius $\varrho$ such that $u_{\varrho}(t, x):=T(t) f_{\varrho}(x) \geq 1-\eta$ for every $t \leq \bar{t}$ and $|x| \leq \varrho_{0}+1$. Consider the function $w(t, x)=(1-\eta)[1-v(t,|x|)]$ for $t \geq 0$ and $|x| \geq \varrho_{0}+1$. It belongs to $C^{1,2}(] 0, \infty\left[\times \mathbb{R}^{N} \backslash \bar{B}_{\varrho_{0}+1}\right) \cap C([0, \infty[\times$ $\left.\mathbb{R}^{N} \backslash B_{\varrho_{0}+1}\right)$. Moreover, $w$ is nondecreasing with respect to $t$ and nonincreasing with respect to $|x|$ and satisfies

$$
\left\{\begin{array}{l}
w(t, x) \leq 1-\eta, \quad t \geq 0, \quad|x| \geq \varrho_{0}+1 \\
w(\bar{t}, x) \geq(1-\eta)^{2}, \quad|x| \geq \varrho_{0}+1
\end{array}\right.
$$

We show that $u_{\varrho}(t, x) \geq w(t, x)$ for $t \leq \bar{t},|x| \geq \varrho_{0}+1$. First of all, observe 
that

$$
\begin{aligned}
D_{t} w(t, x)- & A w(t, x) \\
& =\mathcal{Q}(x)\left[\frac{D_{t} w(t, x)}{\mathcal{Q}(x)}-D_{r r} w(t, x)-\frac{\mathcal{A}(x)-1}{r} D_{r} w(t, x)\right] \\
& \leq \mathcal{Q}(x)\left[\frac{D_{t} w(t, x)}{\mathcal{Q}^{-}(x)}-D_{r r} w(t, x)-\frac{\mathcal{A}^{+}(x)-1}{r} D_{r} w(t, x)\right] \\
& =\frac{\mathcal{Q}(x)}{\mathcal{Q}^{-}(x)}\left[D_{t} w(t, x)-C^{-} w(t, x)\right]=0,
\end{aligned}
$$

since $D_{t} w \geq 0$ and $D_{r} w \leq 0$. As a consequence, the function $h(t, x)=$ $u_{\varrho}(t, x)-w(t, x)$ has the following properties:

$$
\begin{cases}D_{t} h(t, x)-A h(t, x) \geq 0, & t>0,|x| \geq \varrho_{0}+1, \\ h(0, x)=f_{\varrho}(x) \geq 0, & |x| \geq \varrho_{0}+1 \\ h(t, x) \geq 0, & 0 \leq t \leq \bar{t},|x|=\varrho_{0}+1 .\end{cases}
$$

Since $h$ is bounded, it is nonnegative for $t \leq \bar{t},|x| \geq \varrho_{0}+1$, according to Lemma 3.13 below. Therefore, since $f_{\varrho}(x) \leq \chi_{B_{\varrho}}, T(\bar{t}) \chi_{B_{\varrho}}(x)=$ $p\left(\bar{t}, x, B_{\varrho}\right) \geq T(\bar{t}) f_{\varrho}(x)=u_{\varrho}(\bar{t}, x) \geq w(\bar{t}, x) \geq(1-\eta)^{2}=1-\varepsilon$ for $|x| \geq \varrho_{0}+1$. Since also $p\left(\bar{t}, x, B_{\varrho}\right) \geq u_{\varrho}(\bar{t}, x) \geq 1-\varepsilon$ for $|x| \leq \varrho_{0}+1$, the proof is complete.

The following lemma has been used in the proof of the above theorem. It is a parabolic version of Corollary 3.11 in [22].

LEMMA 3.13. Suppose that $\infty$ is inaccessible for $B^{+}$and let $h \in C([0, \tau]$ $\left.\times \mathbb{R}^{N} \backslash B_{\varrho}\right)$ be a bounded function of class $C^{1,2}$ in $\left.] 0, \tau\right] \times \mathbb{R}^{N} \backslash \bar{B}_{\varrho}$, satisfying

$$
\begin{cases}D_{t} h(t, x)-A h(t, x) \geq 0, & t>0,|x|>\varrho, \\ h(0, x) \geq 0, & |x| \geq \varrho, \\ h(t, x) \geq 0, & 0 \leq t \leq \tau,|x|=\varrho .\end{cases}
$$

Then $h \geq 0$.

Proof. We consider a positive $C^{2}$ function $V$ such that $V-A V \geq 0$ in $\mathbb{R}^{N} \backslash B_{\varrho}$ and $V(x) \rightarrow \infty$ as $|x| \rightarrow \infty$ (see the proof of Corollary 3.11 in [22]) and define $h_{\varepsilon}(t, x)=h(t, x)+\varepsilon e^{t} V(x)+\varepsilon t$. Then $D_{t} h_{\varepsilon}-A h_{\varepsilon} \geq \varepsilon$ and $h_{\varepsilon}(t, x) \rightarrow \infty$ as $|x| \rightarrow \infty$, uniformly in $t \in[0, \tau]$, so that $h_{\varepsilon}$ has an absolute minimum at a point $\left(t_{0}, x_{0}\right)$. The case $t_{0}>0$ and $\left|x_{0}\right|>\varrho$ implies $D_{t} h_{\varepsilon}\left(t_{0}, x_{0}\right) \leq 0$ and $A h_{\varepsilon}\left(t_{0}, x_{0}\right) \geq 0$, hence cannot occur because it contradicts the inequality $D_{t}\left(h_{\varepsilon}\right)-A h_{\varepsilon} \geq \varepsilon$. Then necessarily $t_{0}=0$ or $\left|x_{0}\right|=\varrho$ and obviously $h_{\varepsilon}\left(t_{0}, x_{0}\right) \geq 0$. This shows that $h_{\varepsilon} \geq 0$ and, letting $\varepsilon \rightarrow 0, h \geq 0$ as well.

Let us specialise the above result to the case $a_{i j}=\delta_{i j}$. 
Corollary 3.14. Let $A u=\Delta u+\sum_{i=1}^{N} b_{i}(x) D_{i} u(x)$ and suppose that

$$
\frac{1}{r}\left(N-1+\sum_{i=1}^{N} b_{i}(x) x_{i}\right) \leq c(r)
$$

with

$$
\exp \left\{\int_{1}^{r} c(s) d s\right\} \cdot \int_{1}^{r} \exp \left\{-\int_{1}^{s} c(\xi) d \xi\right\} d s \in L^{1}(] 1, \infty[)
$$

Then $(T(t))_{t \geq 0}$ is compact.

Proof. In fact $\infty$ is an entrance boundary for the operator $y^{\prime \prime}+c(r) y^{\prime}$ and hence for $C^{-}$, by Lemma 2.3 .

4. Consequences of compactness. In this section we assume that $(T(t))_{t \geq 0}$ is generated by $\left(A, D_{\max }(A)\right)$ and we draw several consequences of compactness. We start with the following easy result which is not, however, a consequence of the general theory, since the semigroup is not strongly continuous.

Proposition 4.1. If $\lambda-A$ is injective on $D_{\max }(A)$ and $(T(t))_{t \geq 0}$ is compact, then the following statements hold.

(i) The semigroup $(T(t))_{t \geq 0}$ is norm-continuous for $t>0$, that is, $\|T(t+h)-T(t)\| \rightarrow 0$ as $h \rightarrow 0$ for all $t>0$.

(ii) The resolvent $R(\lambda, A)$ is compact.

Proof. (i) We first show that the semigroup is strongly continuous for $t>0$, i.e., for every $t>0$ and $f \in C_{\mathrm{b}}\left(\mathbb{R}^{N}\right),\|T(t+h) f-T(t) f\|_{\infty} \rightarrow 0$ as $h \rightarrow 0$. Fix $f \in C_{\mathrm{b}}\left(\mathbb{R}^{N}\right),\|f\|_{\infty} \leq 1$, and let $\varepsilon>0$ and $\varrho>0$ be such that estimate (3.4) holds. Then

$$
T(t+h) f(x)=\int_{B_{\varrho}} p(t, x, y) T(h) f(y) d y+\int_{\mathbb{R}^{N} \backslash B_{\varrho}} p(t, x, y) T(h) f(y) d y .
$$

Since $T(h) f \rightarrow f$ as $h \rightarrow 0$, uniformly on $\bar{B}_{\varrho}$, we easily deduce from the above equality that $\|T(t+h) f-T(t) f\|_{\infty} \leq 3 \varepsilon$ for $h$ close to 0 .

The full statement now follows from the standard theory of strongly continuous semigroups, as in Theorem 3.2(iv).

(ii) For $f \in C_{\mathrm{b}}\left(\mathbb{R}^{N}\right)$,

$$
R(\lambda, A) f=\int_{0}^{\infty} e^{-\lambda t} T(t) f d t
$$

and by norm-continuity the above integral converges in the operator norm. Therefore, the compactness of $(T(t))_{t \geq 0}$ implies that of $R(\lambda, A)$.

We now prove a partial converse to Theorem 3.12 . 
Theorem 4.2. If $\lambda-A$ is injective on $D_{\max }(A)$ and $R(\lambda, A)$ is compact, then neither $R(\lambda, A)$ nor $(T(t))_{t \geq 0}$ preserves $C_{0}\left(\mathbb{R}^{N}\right)$. Moreover, $\infty$ is an entrance boundary for $C^{+}$.

Proof. For $\varrho>1$, let $f_{\varrho} \in C_{0}\left(\mathbb{R}^{N}\right)$ be such that $\chi_{B_{\varrho-1}} \leq f_{\varrho} \leq \chi_{B_{\varrho}}$ and $u_{\varrho}=R(1, A) f_{\varrho}$. Since $R(1, A)$ is compact and $f_{\varrho} \rightarrow 1$ uniformly on compact sets, as in the proof of Proposition 3.4 we deduce that $u_{\varrho}$ converges uniformly as $\varrho \rightarrow \infty$ to a bounded solution of the equation $u-A u=1$. Since $\lambda-A$ is injective on $D_{\max }(A)$ it follows that $u=1$ and hence $u_{\varrho} \notin C_{0}\left(\mathbb{R}^{N}\right)$ for large $\varrho$. This implies that the resolvent and the semigroup do not preserve $C_{0}\left(\mathbb{R}^{N}\right)$.

We now show that $\infty$ is an entrance boundary for $C^{+}$. Fix $\varrho_{0}$ such that $u_{\varrho_{0}}(x) \geq 1 / 2$ for every $x \in \mathbb{R}^{N}$ and consider a positive decreasing solution $v$ of the problem

$$
\left\{\begin{array}{l}
v-C^{+} v=0, \quad r>\varrho_{0}, \\
v\left(\varrho_{0}\right)=1 .
\end{array}\right.
$$

Then

$$
\begin{aligned}
v-A v & =\mathcal{Q}(x)\left[\frac{v(r)}{\mathcal{Q}(x)}-v^{\prime \prime}(r)-\frac{\mathcal{A}(x)-1}{r} v^{\prime}(r)\right] \\
& \geq \mathcal{Q}(x)\left[\frac{v(r)}{\mathcal{Q}^{+}(x)}-v^{\prime \prime}(r)-\frac{\mathcal{A}^{-}(x)-1}{r} v^{\prime}(r)\right]=\frac{\mathcal{Q}(x)}{\mathcal{Q}^{+}(x)}\left[v-C^{+} v\right]=0
\end{aligned}
$$

for $|x|>\varrho_{0}$ and hence the function $w=v-u_{\varrho_{0}}$ satisfies

$$
\begin{cases}w(x)-A w(x) \geq 0, & |x|>\varrho_{0}, \\ w(x) \geq 0, & |x|=\varrho_{0} .\end{cases}
$$

We show that $w \geq 0$. Suppose that $w<0$ at some point and let $u_{n}$ solve the problem

$$
\begin{cases}u_{n}(x)-A u_{n}(x)=0, & |x|<n \\ u_{n}(x)=-\|w\|_{\infty}, & |x|=n\end{cases}
$$

Then $-\|w\|_{\infty} \leq u_{n} \leq 0$ in $B_{n}$ and the maximum principle applied to $w-u_{n}$ in the annulus $\varrho_{0} \leq|x| \leq n$ yields $u_{n} \leq w$ for $n$ large. Letting $n \rightarrow \infty$ we obtain a bounded solution of the equation $u-A u=0$ such that $u(x) \leq$ $w(x)$ for $|x| \geq \varrho_{0}$, hence different from 0 . Since this cannot happen by the injectivity of $1-A$ on $D_{\max }(A)$, it follows that $w \geq 0$, that is, $v(|x|) \geq$ $u_{\varrho_{0}}(x) \geq 1 / 2$ for $|x| \geq \varrho_{0}$. This shows that no nonzero solution of the equation $v-C^{+} v=0$ tends to 0 as $r \rightarrow \infty$. Theorem 2.2(iii) then implies that $\infty$ is an entrance boundary for $C^{+}$.

REMARK 4.3. We conjecture that the compactness of $R(\lambda, A)$ implies that of $(T(t))_{t \geq 0}$. If $(T(t))_{t \geq 0}$ is compact, then it is easy to see that $L^{p}\left(\mathbb{R}^{N}\right)$ is not preserved under the resolvent and the semigroup. In fact, fix $\varepsilon, \bar{t}>0$ and let $\varrho$ be such that (3.4) holds for every $x \in \mathbb{R}^{N}$ and every $t \geq \bar{t}$ (see 
also Remark 3.7). If $f \in C_{0}\left(\mathbb{R}^{N}\right), f \geq \chi_{B_{\varrho}}$, then $T(t) f(x) \geq 1-\varepsilon$ for every $x \in \mathbb{R}^{N}, t \geq \bar{t}$ and

$$
R(\lambda, A) f(x)=\int_{0}^{\infty} e^{-\lambda t} T(t) f(x) d t \geq \frac{1-\varepsilon}{\lambda} e^{-\lambda \bar{t}} .
$$

The compactness of the semigroup $(T(t))_{t \geq 0}$ gives some information also on its asymptotic behaviour. In order to see this, we recall some basic facts about invariant measures. A probability measure $\mu$ defined on the Borel subsets of $\mathbb{R}^{N}$ is called an invariant measure for $(T(t))_{t \geq 0}$ if for every $f \in$ $C_{\mathrm{b}}\left(\mathbb{R}^{N}\right)$ and $t \geq 0$,

$$
\int_{\mathbb{R}^{N}} T(t) f d \mu=\int_{\mathbb{R}^{N}} f d \mu
$$

An immediate consequence of the existence of an invariant measure is that $T(t) 1=1$ for every $t \geq 0$ and hence $\lambda-A$ is injective on $D_{\max }(A)$ and the generator of $(T(t))_{t \geq 0}$ is $\left(A, D_{\max }(A)\right)$. Since $(T(t))_{t \geq 0}$ is irreducible and has the strong Feller property, from [8, Theorem 4.2.1] it follows that, if an invariant measure exists, it is unique and is also absolutely continuous with respect to the Lebesgue measure. Moreover, its density is a positive function. We refer to [3] for regularity results on densities of invariant measures.

The asymptotic behaviour of $(T(t))_{t \geq 0}$ is clear when an invariant measure $\mu$ exists. Defining the projection $P_{\mu}: C_{\mathrm{b}}\left(\mathbb{R}^{N}\right) \rightarrow C_{\mathrm{b}}\left(\mathbb{R}^{N}\right)$ by

$$
P_{\mu} f(x)=\int_{\mathbb{R}^{N}} f d \mu
$$

for every $f \in C_{\mathrm{b}}\left(\mathbb{R}^{N}\right)$ and $x \in \mathbb{R}^{N}$, one obtains $T(t) f(x) \rightarrow P_{\mu} f(x)$ pointwise as $t \rightarrow \infty$, for every $f \in C_{\mathrm{b}}\left(\mathbb{R}^{N}\right)$ (see [8, Theorem 4.2.1]).

If $(T(t))_{t \geq 0}$ is compact one can prove norm-convergence of $T(t)$ to the projection $P_{\mu}$, as we show below.

Proposition 4.4. Assume that $(T(t))_{t \geq 0}$ is generated by $\left(A, D_{\max }(A)\right)$ and is compact. Then

(i) $(T(t))_{t \geq 0}$ has an invariant measure $\mu$.

(ii) $\left\|T(t)-P_{\mu}\right\| \rightarrow 0$ exponentially as $t \rightarrow \infty$, where $P_{\mu}$ is the projection defined in (4.1).

(iii) There exists a positive $\delta$ such that $\operatorname{Re} \lambda \leq-\delta$ for every $0 \neq \lambda \in$ $\sigma(A)$.

Proof. (i) Fix $\bar{t}>$ 0. From Proposition 3.6 (see also Remark 3.7) we deduce that for every fixed $x_{0} \in \mathbb{R}^{N}$ the family $\left(p\left(t, x_{0}, \cdot\right)\right)_{t \geq \bar{t}}$ of probability measures is tight. Hence a suitable sequence $\left(p\left(t_{n}, x_{0}, \cdot\right)\right)$ with $t_{n} \rightarrow \infty$ converges weakly (with respect to the duality defined by $C_{\mathrm{b}}\left(\mathbb{R}^{N}\right)$ ) to an invariant measure $\mu$ (see [8, Theorem 3.1.1]). 
(ii) Let $G=\operatorname{Ker} P_{\mu}, H=\operatorname{Ker}\left(I-P_{\mu}\right)$ and observe that $G$ and $H$ are $(T(t))_{t \geq 0}$ invariant, that $H$ is one-dimensional and that $C_{\mathrm{b}}\left(\mathbb{R}^{N}\right)$ is the direct sum of $G$ and $H$.

Let us show that the spectral radius of $T(1)_{\mid G}$ is less than 1 . Since $T(1)$ is contractive and compact, it suffices to show that 1 is not an eigenvalue of $T(1)_{\mid G}$. To prove this, assume that $T(1) f=f$ for some $f \in G$. Then $f=T(n) f \rightarrow P_{\mu} f$ pointwise as $n \rightarrow \infty$, hence $f=0$.

From the formula for the spectral radius we obtain

$$
\lim _{n \rightarrow \infty}\left\|T(1)_{\mid G}^{n}\right\|^{1 / n}=\lim _{n \rightarrow \infty}\left\|T(n)_{\mid G}\right\|^{1 / n}<1,
$$

hence $\left\|T(n)_{\mid G}\right\|<1$ for $n$ large and, by the semigroup law, $\left\|T(t)_{\mid G}\right\| \rightarrow 0$ exponentially as $t \rightarrow \infty$. This proves (ii) since $T(t)_{\mid H}$ is the identity.

(iii) $\sigma(A)$ consists of isolated eigenvalues, since $R(\lambda, A)$ is compact, and is given by $\sigma(A)=\sigma\left(A_{\mid G}\right) \cup \sigma\left(A_{\mid H}\right)=\sigma\left(A_{\mid G}\right) \cup\{0\}$. Since $\left\|T(t)_{\mid G}\right\| \rightarrow 0$ exponentially as $t \rightarrow \infty$, there exists $\delta>0$ such that $\operatorname{Re} \lambda \leq-\delta$ for every $\lambda \in \sigma\left(A_{\mid G}\right)$ and (iii) follows.

Since $(T(t))_{t \geq 0}$ is a semigroup of contractions in $C_{\mathrm{b}}\left(\mathbb{R}^{N}\right)$ and $\mu$ is an invariant measure, it is well known that it extends to a strongly continuous semigroup of contractions (still denoted by $(T(t))_{t \geq 0}$ ) in $L^{p}(\mu)$ for every $1 \leq p<\infty$. Its generator $\left(A_{p}, D_{p}\right)$ is an extension of $\left(A, D_{\max }(A)\right)$ as one can deduce from the description of $D_{\max }(A)$ given by (2.3), using dominated convergence.

The asymptotic behaviour of $(T(t))_{t \geq 0}$ in $L^{p}(\mu)$ and some of its spectral properties are easily deduced in the following theorem from the corresponding ones in $C_{\mathrm{b}}\left(\mathbb{R}^{N}\right)$.

Theorem 4.5. If $(T(t))_{t \geq 0}$ is generated by $\left(A, D_{\max }(A)\right)$ and is compact in $C_{\mathrm{b}}\left(\mathbb{R}^{N}\right)$, then the following statements hold for $1<p<\infty$ :

(i) $(T(t))_{t \geq 0}$ is compact in $L^{p}(\mu)$.

(ii) $T(t) \rightarrow P_{\mu}$ exponentially as $t \rightarrow \infty$ in the operator norm of $L^{p}(\mu)$.

(iii) $R\left(\lambda, A_{p}\right)$ is compact in $L^{p}(\mu)$.

(iv) The spectrum of $\left(A_{p}, D_{p}\right)$ consists of isolated eigenvalues and coincides with the spectrum of $\left(A, D_{\max }(A)\right)$. Therefore the spectral gap of Proposition 4.4(iii) holds.

Proof. Since the density of $\mu$ (with respect to the Lebesgue measure) is positive and $(T(t))_{t \geq 0}$ has the strong Feller property, it follows that $T(t)$ maps $L^{\infty}(\mu)$ into $C_{\mathrm{b}}\left(\mathbb{R}^{N}\right)$ for every $t>0$. Moreover, by the semigroup law, it is compact from $L^{\infty}(\mu)$ into $C_{\mathrm{b}}\left(\mathbb{R}^{N}\right)$, hence from $L^{\infty}(\mu)$ into itself. By interpolation (see the next proposition) we deduce that $(T(t))_{t \geq 0}$ is compact in $L^{p}(\mu)$ for $1<p<\infty$. The same property holds for the resolvent, since $(T(t))_{t \geq 0}$ is strongly continuous in $L^{p}(\mu)$. This proves (i) and (iii). 
By interpolation again, we deduce (ii) from Proposition 4.4(ii). Finally, an application of [1, Proposition 2.6] yields (iv).

We now state and prove the version of the interpolation theorem used in the above proof; see [19] where, however, the case $p=\infty$ is excluded. Even though this result is probably known, we give a simple, direct proof.

Proposition 4.6. Let $\mu$ be a probability measure on $\mathbb{R}^{N}$ and $T$ be a compact operator in $L^{\infty}(\mu)$, bounded in $L^{1}(\mu)$. Then $T$ is compact in $L^{p}(\mu)$ for every $1<p<\infty$.

Proof. Let $\mathcal{K}=T(U)$, where $U$ is the unit ball of $L^{\infty}(\mu)$. Fix $\varepsilon>0$ and let $s_{i}(i=1, \ldots, m)$ be simple functions such that the set $\left\{s_{1}, \ldots, s_{m}\right\}$ is an $\varepsilon$-net for $U$. Choose now a partition $\Gamma_{1}, \ldots, \Gamma_{k}$ of $\mathbb{R}^{N}$ of measurable sets having positive measure such that $P_{\varepsilon} s_{i}=s_{i}$ for $i=1, \ldots, m$, where

$$
P_{\varepsilon} f=\sum_{i=1}^{k}\left(\frac{1}{\mu\left(\Gamma_{i}\right)} \int_{\Gamma_{i}} f d \mu\right) \cdot \chi_{\Gamma_{i}} .
$$

It follows that $\left\|P_{\varepsilon} T-T\right\|_{\infty} \leq 2 \varepsilon,\left\|P_{\varepsilon} T-T\right\|_{1} \leq 2\|T\|_{1}$ and hence

$$
\left\|P_{\varepsilon} T-T\right\|_{p} \leq\left(2\|T\|_{1}\right)^{1 / p}\left(\left\|P_{\varepsilon} T-T\right\|_{\infty}\right)^{1-1 / p}
$$

by the Riesz-Thorin interpolation theorem. Letting $\varepsilon \rightarrow 0$ we deduce the compactness of $T$ in $L^{p}(\mu)$, since the operators $P_{\varepsilon} T$ have finite rank.

5. Examples. In this section we present some examples that can be discussed using the results of this paper. We start with the case of radial operators where our conditions become necessary and sufficient for compactness.

EXAMPLE 5.1. Recalling that by radial operators we mean operators $A$ such that $A u$ is independent of $\omega$ if $u$ is so, we notice that from (2.11) it is easily seen that $A$ is radial if and only if the functions $\mathcal{Q}$ and $\mathcal{A}$ are independent of $\omega$. In this case, obviously, all the operators $B^{ \pm}, C^{ \pm}$coincide with $A$ itself. On radial functions they are given by the one-dimensional operator

$$
B y(r):=\mathcal{Q}(r)\left[y^{\prime \prime}(r)+\frac{\mathcal{A}(r)-1}{r} y^{\prime}(r)\right] .
$$

From Propositions 3.1, 3.4 and Theorem 3.2, it follows that $(T(t))_{t \geq 0}$ is generated by $(A, D(A))$ (hence it is compact) if and only if $\infty$ is accessible for $B$. In particular, if $A u=\Delta u+c r^{\gamma} u_{r}$, this happens if and only if $c>0$ and $\gamma>1$.

On the other hand, the generator of $(T(t))_{t \geq 0}$ is $\left(A, D_{\max }(A)\right)$ if and only if $\infty$ is inaccessible for $B$ and, in this case, $(T(t))_{t \geq 0}$ is compact if and only if $\infty$ is an entrance boundary for $B$; see Theorems 3.12, 4.2. If $A u=\Delta u+c r^{\gamma} u_{r}$, then the generator is $\left(A, D_{\max }(A)\right)$ if and only if $c \leq 0$ or 
$c>0, \gamma \leq 1$, and moreover, $(T(t))_{t \geq 0}$ is compact if and only if $c<0, \gamma>1$. We also refer to Examples 7.3 and 7.4 in [22] where wider classes of radial operators are discussed.

For one-dimensional operators the results of this paper can be generalised to the case of arbitrary intervals.

EXAMPLE 5.2. Let

$$
A y=a\left(y^{\prime \prime}+b y^{\prime}\right)
$$

on a (possibly unbounded) open interval $I=] r_{1}, r_{2}[$. By a straightforward generalisation of the proofs of Theorems 3.2, 3.12, 4.2 and Proposition 3.4, we conclude that $(T(t))_{t \geq 0}$ is generated by $(A, D(A))$ and is compact if and only if both $r_{1}, r_{2}$ are accessible boundaries, whereas $(T(t))_{t \geq 0}$ is generated by $\left(A, D_{\max }(A)\right)$ and is compact if and only if both $r_{1}, r_{2}$ are entrance boundaries.

Let us now analyse some nonradial situations.

ExAmple 5.3. Consider operators $A$ as in (1.1) assuming that the matrix $\left(a_{i j}\right)$ is uniformly elliptic. In this case, Proposition 3.1 implies that the generator of $(T(t))_{t \geq 0}$ is $(A, D(A))$, hence $(T(t))_{t \geq 0}$ is compact, provided that

$$
\sum_{i=1}^{N} b_{i}(x) x_{i} \geq \beta|x|^{2+\gamma}
$$

for some $\beta, \gamma>0$ and $|x|$ large. On the other hand, if there are positive $\beta, \gamma$ such that, for large $|x|$,

$$
\sum_{i=1}^{N} b_{i}(x) x_{i} \leq-\beta|x|^{2+\gamma},
$$

then $(T(t))_{t \geq 0}$ is generated by $\left(A, D_{\max }(A)\right)$ and is compact, by Corollary 3.11 .

In the following example we show that if the diffusion matrix grows at most quadratically and the radial drift at most linearly, then the semigroup is not compact in $C_{\mathrm{b}}\left(\mathbb{R}^{N}\right)$.

EXAmPle 5.4. Consider operators $A$ as in (1.1) satisfying the condition

$$
\sum_{i=1}^{N} a_{i i}(x)+\left|\sum_{i=1}^{N} b_{i}(x) x_{i}\right| \leq C\left(1+|x|^{2}\right)
$$

for some $C>0$. From Proposition 3.5 we deduce that $\left(A, D_{\max }(A)\right)$ is the generator of $(T(t))_{t \geq 0}$. Since the semigroup preserves $C_{0}\left(\mathbb{R}^{N}\right)$ (see $[22$, Corollary 3.18]), from Theorem 4.2 it follows that neither the semigroup nor the resolvent is compact. 
Particular cases of the above situation are uniformly elliptic operators and the Ornstein-Uhlenbeck operator

$$
A=\sum_{i, j=1}^{N} q_{i j} D_{i j}+\sum_{i, j=1}^{N} b_{i j} x_{j} D_{i},
$$

where $Q=\left(q_{i j}\right)$ is a real, symmetric and positive definite matrix and $B=$ $\left(b_{i j}\right)$ is a nonzero real matrix. It should be noted, however, that if $\operatorname{Re} \lambda<0$ for every eigenvalue of the matrix $B$, then the Ornstein-Uhlenbeck semigroup has an invariant measure $\mu$ and it is compact in $L^{p}(\mu)$ for $1<p<\infty$ (see [9] and [6]). This shows that Theorem 4.5 yields only sufficient conditions for compactness in $L^{p}(\mu)$.

In the above examples the compactness of $(T(t))_{t \geq 0}$ is a consequence of the growth (at infinity) of the vector field $b=\left(b_{1}, \ldots, b_{N}\right)$. It is worth mentioning that the semigroup can be compact even though $b=0$.

EXAMPLE 5.5. Writing a point $x \in \mathbb{R}^{N}$ in the form $x=r \omega$, we consider an operator $A$ as in (1.1) such that $A u=\Delta u+r^{\gamma} D_{r r} u$ for $r \geq 1$, with $\gamma>2$. Clearly $b=0$ and $a_{i j}(x)=1+r^{\gamma} \omega_{i} \omega_{j}$ for $r \geq 1$. The matrix $\left(a_{i j}(x)\right)$ has eigenvalues 1 with multiplicity $N-1$ and $1+r^{\gamma}$ with multiplicity 1 and eigenvector $\omega$. It follows that $\sum_{i=1}^{N} a_{i i}(x)-2 \mathcal{Q}(x)=N-2-|x|^{\gamma}$ and hence Corollary 3.11 shows that $(T(t))_{t \geq 0}$ is generated by $\left(A, D_{\max }(A)\right)$ and is compact.

In the following two-dimensional examples the combination of a strong radial drift outside an angle $S$ and of a tangential drift in $S$ yields the compactness of $(T(t))_{t \geq 0}$. The domain of the generator depends on the sign of the radial drift.

We use polar coordinates $r, \vartheta$, identifying the point $x \in \mathbb{R}^{2}$ with the complex number $r e^{i \vartheta}$. For $\alpha>0$, let $S$ be the angle $S:=\{r \geq 0,0 \leq \vartheta \leq \alpha\}$. Let moreover $\varphi$ be any smooth function such that $0 \leq \varphi \leq 1, \varphi(\vartheta)=0$ for $\vartheta \notin] 0, \alpha[$ and $\varphi(\vartheta)>0$ for $\vartheta \in] 0, \alpha[$.

EXAmPle 5.6. Consider the operator

$$
A u:=\Delta u-\varphi(\vartheta) r^{\tau-1} u_{\vartheta}-(1-\varphi(\vartheta)) r^{\sigma} u_{r}
$$

with $\sigma, \tau>1$. Taking $V(r, \vartheta)=h(\vartheta) \log r$ (with $r \geq 2$ and $h$ being $2 \pi$ periodic) we obtain

$$
A V(r, \vartheta)=h^{\prime \prime}(\vartheta) r^{-2} \log r-\varphi(\vartheta) h^{\prime}(\vartheta) r^{\tau-1} \log r-(1-\varphi(\vartheta)) h(\vartheta) r^{\sigma-1} .
$$

Choosing $h$ positive and such that $h^{\prime}(\vartheta)>0$ if $\vartheta \in[0, \alpha]$ we see that $A V \leq$ $c_{1}-c_{2} V^{2}$ for some $c_{1}, c_{2}>0$. Theorem 3.10 shows that $(T(t))_{t \geq 0}$ is generated by $\left(A, D_{\max }(A)\right)$ and is compact. 


\section{EXAMPle 5.7. Consider now}

$$
A u:=\Delta u-\varphi(\vartheta) r^{\tau-1} u_{\vartheta}+(1-\varphi(\vartheta)) r^{\sigma} u_{r}
$$

with $\sigma, \tau>1$ and the function $V(r, \vartheta)=h(\vartheta) / \log r$ (with $r \geq 1$ ), where $h$ is as in Example 5.6. Then $\Delta V$ is bounded and

$$
A V(r, \vartheta)=\Delta V-\varphi(\vartheta) h^{\prime}(\vartheta) r^{\tau-1}(\log r)^{-1}-(1-\varphi(\vartheta)) h(\vartheta) r^{\sigma-1}(\log r)^{-2}
$$

diverges to $-\infty$ as $r \rightarrow \infty$. From Proposition 3.1 we deduce that $\widehat{D}=D(A)$ and hence the semigroup is compact.

\section{References}

[1] W. Arendt, Gaussian estimates and interpolation of the spectrum in $L^{p}$, Differential Integral Equations 7 (1994), 1153-1168.

[2] R. Azencott, Behaviour of diffusion semi-groups at infinity, Bull. Soc. Math. France 102 (1974), 193-240.

[3] V. Bogachev, N. I. Krylov and M. Röckner, On regularity of transition probabilities and invariant measures of singular diffusions under minimal conditions, Comm. Partial Differential Equations 26 (2001), 2037-2080.

[4] S. Cerrai, A Hille-Yosida theorem for weakly continuous semigroups, Semigroup Forum 49 (1994), 349-367.

[5] - Second Order PDE's in Finite and Infinite Dimension, Lecture Notes in Math. 1762, Springer, 2001.

[6] A. Chojnowska-Michalik and B. Gołdys, Existence, uniqueness and invariant measures for stochastic semilinear equations on Hilbert spaces, Probab. Theory Related Fields 102 (1995), 331-356.

[7] G. Da Prato and B. Gołdys, Elliptic operators on $\mathbb{R}^{d}$ with unbounded coefficients, J. Differential Equations 172 (2001), 333-358.

[8] G. Da Prato and J. Zabczyk, Ergodicity for Infinite Dimensional Systems, Cambridge Univ. Press, 1996.

[9] —, - Regular densities of invariant measures in Hilbert spaces, J. Funct. Anal. 130 (1995), 427-449.

[10] E. B. Davies, $L^{1}$ properties of second order elliptic operators, Bull. London Math. Soc. 17 (1985), 417-436.

[11] K.-J. Engel and R. Nagel, One-Parameter Semigroups for Linear Evolution Equations, Grad. Texts in Math. 194, Springer, 2000.

[12] S. N. Ethier and T. G. Kurtz, Markov Processes. Characterization and Convergence, Wiley, 1986.

[13] W. Feller, The parabolic differential equations and the associated semi-groups of transformations, Ann. of Math. 55 (1952), 468-519.

[14] -, Diffusion processes in one dimension, Trans. Amer. Math. Soc. 77 (1954), 1-31.

[15] A. Friedman, Partial Differential Equations of Parabolic Type, Prentice-Hall, 1964.

[16] D. Gilbarg and N. Trudinger, Elliptic Partial Differential Equations of Second Order, Grundlehren Math. Wiss. 224, Springer, 1983.

[17] R. Z. Has'minskiŭ, Ergodic properties of recurrent diffusion processes and stabilization of the solution to the Cauchy problem for parabolic equations, Theory Probab. Appl. 5 (1960), 179-196. 
[18] R. Z. Has'minskiǔ, Stochastic Stability of Differential Equations, Nauka, 1969 (in Russian); English transl.: Sijthoff and Noordhoff, 1980.

[19] M. A. Krasnosel'skiı̌, On a theorem of M. Riesz, Dokl. Akad. Nauk. SSSR 27 (1960), 246-248 (in Russian); English transl.: Soviet Math. Dokl. 1 (1960), 219-221.

[20] A. Lunardi, Analytic Semigroups and Optimal Regularity in Parabolic Problems, Progr. Nonlinear Differential Equations Appl. 16, Birkhäuser, 1995.

[21] G. Metafune, $L^{p}$ spectrum of Ornstein-Uhlenbeck operators, Ann. Scuola Norm. Sup. Pisa Cl. Sci. (4) 30 (2001), 97-124.

[22] G. Metafune, D. Pallara and M. Wacker, Feller semigroups in $\mathbb{R}^{N}$, Semigroup Forum, to appear.

[23] N. Meyers and J. Serrin, The exterior Dirichlet problem for second-order elliptic partial differential equations, J. Math. Mech. 9 (1960), 513-538.

[24] J. M. A. M. van Neerven and J. Zabczyk, Norm discontinuity of Ornstein-Uhlenbeck semigroups, Semigroup Forum 59 (1999), 389-403.

[25] E. Priola, On a class of Markov type semigroups in uniformly continuous and bounded function spaces, Studia Math. 136 (1999), 271-295.

[26] D. W. Stroock and S. R. S. Varadhan, Multidimensional Diffusion Processes, Springer, 1979.

Dipartimento di Matematica "Ennio De Giorgi"

Università di Lecce

C.P. 193

73100 Lecce, Italy

E-mail: giorgio.metafune@unile.it

pallara@le.infn.it

Mathematisches Institut Universität Tübingen

Auf der Morgenstelle 10 72076 Tübingen, Germany

E-mail: mwacker@wsi.gris.uni-tuebingen.de

Received October 11, 2001

Revised version February 20, 2002 